\title{
Identification of a novel inhibitor of SARS-CoV-2 3CL-PRO through virtual screening and molecular dynamics simulation
}

\author{
Asim Kumar Bepari ${ }^{\text {Corresp., } 1}$, Hasan Mahmud Reza ${ }^{1}$ \\ ${ }^{1}$ Department of Pharmaceutical Sciences, North South University, Dhaka, Bangladesh \\ Corresponding Author: Asim Kumar Bepari \\ Email address: asim.bepari@northsouth.edu
}

Background. The COVID-19 pandemic, caused by the SARS-CoV-2 virus, has ravaged lives across the globe since December 2019, and new cases are still on the rise. Peoples' ongoing sufferings trigger scientists to develop safe and effective remedies to treat this deadly viral disease. While repurposing the existing FDA-approved drugs remains in the front line, exploring drug candidates from synthetic and natural compounds is also a viable alternative. This study employed a comprehensive computational approach to screen inhibitors for SARS-CoV-2 3CL-PRO (also known as the main protease), a prime molecular target to treat coronavirus diseases.

Methods. We performed 100 ns GROMACS molecular dynamics simulations of three high-resolution xray crystallographic structures of 3CL-PRO. We extracted frames at $10 \mathrm{~ns}$ intervals to mimic conformational diversities of the target protein in biological environments. We then used AutoDock Vina molecular docking to virtual screen the Sigma-Aldrich MyriaScreen Diversity Library II, a rich collection of 10,000 druglike small molecules with diverse chemotypes. Subsequently, we adopted in silico computation of physicochemical properties, pharmacokinetic parameters, and toxicity profiles. Finally, we analyzed hydrogen bonding and other protein-ligand interactions for the short-listed compounds.

Results. Over the 100 ns molecular dynamics simulations of 3CL-PRO's crystal structures, 6LZE, 6M0K, and 6YB7, showed overall integrity with mean $C \alpha$ root-mean-square deviation (RMSD) of 1.96 ( \pm 0.35$) \AA$, $1.98( \pm 0.21) \AA$, and $1.94( \pm 0.25) \AA$, respectively. Average root-mean-square fluctuation (RMSF) values were $1.21 \pm 0.79$ (6LZE), $1.12 \pm 0.72$ (6MOK), and $1.11 \pm 0.60$ (6YB7). After two phases of AutoDock Vina virtual screening of the MyriaScreen Diversity Library II, we prepared a list of the top 20 ligands. We selected four promising leads considering predicted oral bioavailability, druglikeness, and toxicity profiles. These compounds also demonstrated favorable protein-ligand interactions. We then employed 50-ns molecular dynamics simulations for the four selected molecules and the reference ligand 11a in the crystallographic structure 6LZE. Analysis of RMSF, RMSD, and hydrogen bonding along the simulation trajectories indicated that S51765 would form a stable protein-ligand complexe with 3CL-PRO compared to other molecules. Insights into short-range Coulombic and Lennard-Jones potentials also revealed favorable binding of S51765 with 3CL-PRO.

Conclusion. We identified a potential lead for antiviral drug discovery against the SARS-CoV-2 main protease. Our results will aid global efforts to find safe and effective remedies for COVID-19. 
1 Identification of a novel inhibitor of SARS-CoV-2 3CL-PRO

2 through virtual screening and molecular dynamics

3 simulation

Corresponding Author:

10 Asim Bepari ${ }^{1}$

Department of Pharmaceutical Sciences, North South University, Dhaka, Bangladesh Email address: asim.bepari@northsouth.edu

\section{Abstract}

Background. The COVID-19 pandemic, caused by the SARS-CoV-2 virus, has ravaged lives across the globe since December 2019, and new cases are still on the rise. Peoples' ongoing sufferings trigger scientists to develop safe and effective remedies to treat this deadly viral disease. While repurposing the existing FDA-approved drugs remains in the front line, exploring drug candidates from synthetic and natural compounds is also a viable alternative. This study employed a comprehensive computational approach to screen inhibitors for SARS-CoV-2 3CLPRO (also known as the main protease), a prime molecular target to treat coronavirus diseases.

Methods. We performed 100 ns GROMACS molecular dynamics simulations of three highresolution x-ray crystallographic structures of 3CL-PRO. We extracted frames at 10 ns intervals to mimic conformational diversities of the target protein in biological environments. We then used AutoDock Vina molecular docking to virtual screen the Sigma-Aldrich MyriaScreen Diversity Library II, a rich collection of 10,000 druglike small molecules with diverse chemotypes. Subsequently, we adopted in silico computation of physicochemical properties, pharmacokinetic parameters, and toxicity profiles. Finally, we analyzed hydrogen bonding and other protein-ligand interactions for the short-listed compounds.

Results. Over the 100 ns molecular dynamics simulations of 3CL-PRO's crystal structures, 6LZE, 6M0K, and 6YB7, showed overall integrity with mean $\mathrm{C} \alpha$ root-mean-square deviation (RMSD) of $1.96( \pm 0.35) \AA, 1.98( \pm 0.21) \AA$, and $1.94( \pm 0.25) \AA$, respectively. Average rootmean-square fluctuation (RMSF) values were $1.21 \pm 0.79$ (6LZE), $1.12 \pm 0.72$ (6M0K), and 1.11 \pm 0.60 (6YB7). After two phases of AutoDock Vina virtual screening of the MyriaScreen Diversity Library II, we prepared a list of the top 20 ligands. We selected four promising leads considering predicted oral bioavailability, druglikeness, and toxicity profiles. These compounds also demonstrated favorable protein-ligand interactions. We then employed 50-ns molecular 
39

40

41

42

43

44

45

46

47

48

49

50

51

52

53

54

55

56

57

58

59

60

61

62

63

64

65

66

67

68

69

70

71

72

73

74

75

76

77

simulation trajectories indicated that S51765 would form a stable protein-ligand complexe with 3CL-PRO compared to other molecules. Insights into short-range Coulombic and Lennard-Jones potentials also revealed favorable binding of S51765 with 3CL-PRO.

Conclusion. We identified a potential lead for antiviral drug discovery against the SARS-CoV-2 main protease. Our results will aid global efforts to find safe and effective remedies for COVID19.

\section{Introduction}

The "severe acute respiratory syndrome coronavirus 2" (SARS-CoV-2), responsible for the coronavirus disease-2019 (COVID-19), originated in Wuhan, China in late 2019 as a pneumonia outbreak causing acute respiratory distress syndrome and related complications (Huang et al., 2020; Zhou et al., 2020; Wu et al., 2020; Gorbalenya et al., 2020). Considering the severity of symptoms among the affected people and rapid spread, the World Health Organization (WHO) declared COVID-19 as a pandemic on 11 March 2020. This catastrophe has created an unprecedented healthcare crisis confounded with multifaceted economic, social, and cultural impacts (Sultana et al., 2020; McKibbin \& Fernando, 2020; Hartley \& Perencevich, 2020; Headey et al., 2020; Forster et al., 2020). Despite extensive measures taken at individual to global scales, the world has only a few arsenals to fight against this massive disaster. While remdesivir, the only FDA-approved drug to treat COVID-19, is indicated for patients 12 years of age and older requiring hospitalization, we all are in pursuit of safer and more effective antiviral agents.

SARS-CoV-2 virus is closely related to other coronaviruses, including SARS-CoV and MERS$\mathrm{CoV}$, and carries a single-stranded RNA genome of $\sim 30 \mathrm{~kb}$, which encodes at least 14 openreading frames (ORFs) (Zhou et al., 2020; Wu et al., 2020; Kim et al., 2020; Gordon et al., 2020). ORF1a and ORF1ab produce polypeptides pp1a and pp1ab, respectively, which generate nonstructural proteins (nsps) upon proteolytic cleavage and form the replicase-transcriptase complex (Kim et al., 2020; Gordon et al., 2020; Jiang et al., 2020). The activity of 3CL-PRO (also known as 3C-like proteinase, main protease, and $\mathrm{M}^{\mathrm{pro}}$ ) is crucial in the auto-proteolysis of viral polypeptides and is a prime target in the discovery of antiviral agents for COVID-19 (Ziebuhr, Snijder \& Gorbalenya, 2000; Anand et al., 2003; Zhang et al., 2020; Jin et al., 2020).

Many high-resolution x-ray crystallographic structures of SARS-CoV-2 3CL-PRO, in both bound and unbound states, are available in the Protein Data Bank (PDB) (www.wwpdb.org). These three-dimensional structures can significantly help design, discover, and develop potential inhibitors for future therapeutic applications. Computational methods are introducing many quick and efficient avenues to reach destinations in the journey of drug discovery and development (Kapetanovic, 2008; Macalino et al., 2015; Yu \& MacKerell, 2017; Cui et al., 2020). It is noteworthy that proteins are dynamic in a biological environment, in contrast to the static x-ray crystallographic structures. Virtual screening methods for approved drugs or large databases such as ZINC15 usually involve only a few target structures; therefore, they are more likely to leave off potential ligands. In this study, we have employed a comprehensive in silico approach to identify leads for the treatment of COVID-19 through inhibition of the viral main protease. We 
78

79

80

81

82

83

84

85

86

87

88

89

90

91

92

93

94

95

96

97

98

99

100

101

102

103

104

105

106

107

108

109

110

111

112

113

114

115

generated multiple target structures through molecular dynamics simulations of 3CL-PRO crystal structures and performed target-based virtual screening of the MyriaScreen Diversity Library II. Top compounds were then scrutinized for physicochemical properties, pharmacokinetic profiles, and toxicity risks. Subsequently, we performed protein-ligand interaction analyses for the best picks. Results from this comprehensive computational analysis may assist in finding an effective therapeutic intervention for COVID-19.

\section{Materials \& Methods}

\section{Protein Structure}

We retrieved X-ray crystallographic protein structures with PDB IDs 6LZE (Dai et al., 2020), 6M0K (Dai et al., 2020), and 6YB7 from the Protein Data Bank (www.rcsb.org). A multiple structure alignment was done using the mTm-align webserver (Dong et al., 2018a).

\section{Ligand Libraries}

MyriaScreen Diversity Library II is a powerful resource for lead discovery ("Screening Compounds,” 2020). Upon request to Sigma-Aldrich, we received an sdf file of this library which contains 10,000 high-purity screening compounds. Sigma-Aldrich constructed this popular library from over 300,000 compounds on the basis of diversity and drug-likeness. All structures were edited using Open babel (O’Boyle et al., 2011) and Discovery Studio Visualizer (BIOVIA, Dassault Systèmes, Discovery Studio Visualizer, v20.1.0.192, 2019).

\section{Virtual Screening}

All non-amino acid residues from a protein structure were removed using UCSF Chimera alpha version 1.14 (2019) (Pettersen et al., 2004). Then the Dock Prep tool of the Chimera program was used to prepare the protein for docking. All default parameters were selected and the structure was saved as a pdb file. In AutoDockTools version 1.5.6 (Morris et al., 2009) the pdb file was then edited by adding polar hydrogens, merging non-polar hydrogens and adding Kollman charges. The final macromolecule was saved in the pdbqt format.

We used POAP (Parallelized Openbabael and Autodock suite Pipeline) to automate the AutoDock Vina virtual screening process (Samdani \& Vetrivel, 2018). The Ligand Preparation Module of POAP prepared the ligands by adding hydrogens, generating 3D coordinates and minimizing energy. Ligand files were saved in the pdbqt format. Then we used the Virtual screening Module of POAP to screen the ligands using AutoDock Vina (Trott \& Olson, 2010). The inhibitor 11a complexed with 6LZE was used as a guide to make the grid box. For the grid box, the spacing was set at default $1 \AA$, center xyz coordinates were $10.700,0.784,23.667$, and the dimension was $26 \times 26 \times 26$. Exhaustiveness was set at eight. Ligands were ranked based on the binding energy ( $\mathrm{kcal} / \mathrm{mol})$. A more negative value indicates stronger protein-ligand binding.

We performed rigid docking for the best four ligands and the reference inhibitor 11a using AutoDock4.2 (Morris et al., 2009). We used the same ligand and protein files prepared for the Vina virtual screening. For the grid parameter file (.gpf), atom types were selected from the ligands files, the grid was centered on the ligand, grid dimension was $60 \times 60 \times 60$, and the 
116 spacing was $0.375 \AA$. The Lamarckian Genetic Algorithm (LGA) was used for the simulation and 117 the maximum number of energy evaluations was 2,500,000. The best docked poses were selected

118 based on the binding scores and complexes were generated. Subsequently, we used those

119 complexes for protein-ligand interaction analyses in Discovery Studio Visualizer.

\section{Molecular Dynamics Simulations}

121 Molecular dynamics (MD) simulations were carried out using GROMACS (Berendsen, van der

122 Spoel \& van Drunen, 1995; Abraham et al., 2015; Lindahl et al., 2019) and a high-performance

123 computing system equipped with an Intel Xeon CPU and an NVIDIA Tesla K40c GPU. For the

124 best four ligands, we used the protein-ligand complexes generated in AutoDock4.2 docking. For

125 the reference complex 6LZE-11, we used the PDB structure. Protein topologies were prepared by

126 the pdb2gmx module of GROMACS using the CHARMM36 all-atom force field

127 (Vanommeslaeghe et al., 2010) and the TIP 3-point water model. Ligand topologies were

128 generated by the CHARMM General Force Field (CGenFF) program version 2.4.0 ("CGenFF

129 Home”). A dodecahedron box was defined where the protein was positioned at least $1.0 \mathrm{~nm}$ from

130 the box edge, filled with approximately 20,000 water molecules, and four sodium ions were

131 added to neutralize the overall charge. The simulation system was energy minimized with a

132 maximum 50,000 steps of steepest descent minimization algorithm. The solvent and ions were

133 equilibrated in two restrained phases. The reference temperature was $300 \mathrm{~K}$ for the NVT

134 (isothermal-isochoric) ensemble and the reference pressure was 1.0 bar for the subsequent NPT

135 (isothermal-isobaric) ensemble. Finally, we performed unrestrained MD simulations of the

136 equilibrated systems. Leap-frog integrator was used with a step size of 2 fs. Constraint algorithm

137 was LINCS for NVT, NPT, and the production MD runs. The short-range van der Waals cutoff

138 was $1.2 \mathrm{~nm}$. Modified Berendsen thermostat was used for temperature coupling and Parrinello-

139 Rahman barostat was used for pressure coupling. Similar MD parameters were also used in other

140 studies (Selvaraj et al., 2020; Joshi et al., 2020).

141 We analyzed simulation trajectories using the GROMACS analysis tools. We also used VMD

142 (Humphrey, Dalke \& Schulten, 1996) for analyzing protein-ligand hydrogen bonding.

\section{MMPBSA Binding Energy Calculation}

144 Binding free energy for protein-ligand complexes was computed using the g_mmpbsa tool

145 (Kumari et al., 2014). We calculated free energy from MD trajectories separately on two periods, 146 20-25 ns and 45-50 ns, by sampling snapshots at every 100 ps. The binding free energy was

147 calculated as the sum of van der Waal energy, electrostatic energy, polar solvation energy, and

148 the solvent accessible surface area (SASA) energy.

\section{Results}

\section{Molecular Dynamics Simulations of SARS-CoV-2 3CL-PRO}

151 To predict the dynamics and stability of SARS-CoV-2 3CL-PRO, we performed GROMACS 152 molecular dynamics (MD) simulations of three high-resolution structures with PDB IDs 6LZE

153 (1.5 $\AA$ ), 6M0K (1.5 $\AA$ ), and 6YB7 (1.25 $\AA$ ). 6YB7 represents an apo form with unliganded active 
154 sites, whereas 6LZE and 6M0K are holo forms complexed with inhibitors 11a and 11b,

155 respectively. Visualization and alignment indicated significant agreement among the structures

156 (Fig. 1A) with an average pairwise RMSD of 0.52 angstroms and a TM-score of 0.985 (on a scale

157 of 0 to 1). A protein chain was isolated from the complex, and the topology was prepared using

158 the CHARMM-36 force field. The protein was solvated in a water box with appropriate ions to

159 simulate the biological system. The system's potential energy converged very quickly, within

1601000 steps (Fig. 1B), to relax the protein-water system by eliminating unusual steric clashes.

161 During NVT (constant number of particles, volume, and temperature) equilibration, the

162 temperature reached 300K before 10 ps and was maintained (Fig. 1C). Subsequently, the system

163 underwent an equilibration at an NPT (isothermal-isobaric) ensemble, where the system pressure

164 plateaued at 1 bar with some fluctuations (Fig. 1D). These results indicated that the simulation

165 system was well prepared, albeit some minor variations, for the selected protein structures.

166 Next, we proceeded with the 100 ns production MD simulations and the output trajectories were

167 analyzed for various features of the simulation. Visual inspection of frames extracted at different

168 time intervals provides an idea of the dynamics the protein is undergoing in a biological system.

169 For instance, Fig. 1E-J show orientations of the residues GLY143, CYS145, HIS164, and

170 GLU166, which play critical roles in inhibitor binding, at 20 ns intervals. Changes were apparent

171 for GLU166, compared to other labeled residues. Presumably, conformational alterations are

172 apparent for loop regions. We calculated the root-mean-square deviation (RMSD) of all C $\alpha$ atoms

173 in the trajectory in reference to the alpha carbons of energy minimized proteins (Fig. $1 \mathrm{~K}$ ).

174 Average RMSD values for 6LZE, 6M0K, and 6YB7 were $1.96( \pm 0.35) \AA, 1.98( \pm 0.21) \AA$, and

$1751.94( \pm 0.25) \AA$, respectively, indicating overall stability. We also calculated the root-mean-

176 square fluctuation (RMSF), a measure of standard deviations of atomic positions in the trajectory

177 from the reference frames, for the C $\alpha$ domains (Fig. 1L). RMSF values rarely crossed $2 \AA$ for

178 most of the atoms. Mean ( $\pm \mathrm{SD}) \mathrm{RMSF}$ values were $1.21 \pm 0.79$ (6LZE), $1.12 \pm 0.72(6 \mathrm{M} 0 \mathrm{~K})$,

179 and $1.11 \pm 0.60$ (6YB7). We observed very high fluctuations at extreme ends, which is a usual

180 phenomenon. For 6LZE, there is also a spike for atom numbers 567-797, corresponding to the

181 residues from 44 to 53. Again, this is an expected behavior for a protein's loop regions (Fig. $1 \mathrm{~L}$,

182 inset).

183 Together, our results from molecular dynamics simulations infer integrity of SARS-CoV-2 3CL-

184 PRO crystal structures. Nevertheless, the conformations showed some alterations over the $100 \mathrm{~ns}$

185 simulation period, which could have significant biological implications in protein-ligand

186 interactions.

187 AutoDock Vina Virtual Screening of the MyriaScreen Diversity Library II

188 MyriaScreen Diversity Library II comprises 10,000 high-purity compounds suitable for lead

189 discovery. In the first phase, we screened the whole library with the virtual screening module of

190 POAP using AutoDock Vina against three crystal structures, 6LZE, 6M0K, and 6YB7, of 3CL-

191 PRO. Filtering with an average predicted binding affinity of $-8 \mathrm{kcal} / \mathrm{mol}$ or lower generated a

192 combined top list of 286 ligands.

193 We extracted frames at 10 ns intervals from the MD simulation trajectories of 6LZE, 6M0K, and 194 6YB7, which yielded 30 pdb files for the second screening phase. Plus, we included three crystal

Peer] reviewing PDF | (2020:10:54273:2:0:NEW 20 Mar 2021) 
195

196

197

198

199

200

201

202

203

204

205

206

207

208

209

210

211

212

213

214

215

216

217

218

219

220

221

222

223

224

225

226

227

228

229

230

231

232

233

234

235

structures and performed Vina molecular docking to virtual screen the top 286 compounds against 33 target structures for 3CL-PRO. The top 20 compounds based on overall binding affinities are listed in Table 1. The first molecule, R897698, and the last molecule, R461083, showed binding affinities of -8.7 and $-8.0 \mathrm{kcal} / \mathrm{mol}$, respectively. We observe considerable deviations in binding affinities among the 33 protein structures for individual small molecules. Overall, top 20 ligands showed greater affinities to 6LZE compared to other structures (Table 1).

The predictive performance in virtual screening can vary greatly depending on many factors including the target structure, the docking tool, and the docking protocol. We used AutoDock Vina, a free, open source, widely cited, and one of the most efficient docking tools (Durrant et al., 2013; Wang et al., 2016). To validate the screening protocol, we separated the co-crystallized ligand 11a from the PDB structure 6LZE and then re-docked using the same protocol employed for the virtual screening. We superimposed the docked complex to the crystal structure in Pymol. Indeed, the binding pose generated by Vina was a close match with the crystal structure (Fig S1).

For further validation of the Vina virtual screening protocol, we retrieved 50 decoys from the DUD-E database (Mysinger et al., 2012) by supplying 11a as the active ligand. We compared binding scores of 11a, the top 20 hits from the two phase of Vina screening, and 50 decoys (Table S1). Interestingly, when we considered all 33 protein structures, all of the top 20 ligands had superior average scores than the decoys. The reference molecule scored better than most of the decoys, although, nine of the 50 decoys topped 11a by slight margins. To the contrary, 19 decoys scored higher than 11a when we considered only the 6LZE crystal structure. Therefore, our virtual screening protocol seemed to produce reasonable predictive power for the selected ligands and target structures of 3CL-PRO.

\section{In silico ADME/Tox Profiling}

We predicted pharmacokinetic parameters of small molecules through the SwissADME webserver (Daina, Michielin \& Zoete, 2017). Table 2 shows physicochemical and solubility descriptors for the top 20 ligands. Molecular weight varied between 367 to $525 \mathrm{~g} / \mathrm{mol}$, which falls within the optimum range (200-600 g/mol) for druglikeness. The number of rotatable bonds indicates a structure's flexibility, and compounds with 10 or fewer rotatable bonds are considered candidates for good oral bioavailability in rats . Khanna and Ranganathan (2009) showed that the mean number of rotatable bonds was seven for drugs and three for toxins (Khanna \& Ranganathan, 2009). We found the number of rotatable bonds in the range of 0-7 for the top ligands (Table 2). The numbers of H-bond acceptors and donors were 3-9 and 0-4, respectively. The topological polar surface area (TPSA) values are based on the polar fragments' surface contributions and indicate the overall polarity of a compound. Table 2 demonstrates that TPSA values were relatively higher for most of the top-ranked ligands, highest for \#2 and lowest for \#19. Lipophilicity, usually expressed as LogPo/w, is a crucial determinant of a drug's pharmacokinetic and pharmacodynamic profiles. There are different methods for the prediction of LogP. Table 2 displays WLOGP (Wildman \& Crippen, 1999) and consensus LogPo/w of our virtual screening's top compounds. A drug's solubility is better when LogP is less than three, whereas a LogP in the range of -1 to 5.9 enhances membrane permeability (Arnott \& Planey, 2012). All compounds in our list conform to the requirements for lipid solubility. Table 2 also

PeerJ reviewing PDF | (2020:10:54273:2:0:NEW 20 Mar 2021) 
236 demonstrates ESOL LogS values and solubility categories for the top list. The minimum and 237 maximum LogS values were -7.57 and -3.77 for ligand \#16 and \#9, respectively. ESOL estimates 238 the aqueous solubility of a lead directly from the chemical structure (Delaney, 2004). Thus, 239 ligand \#9 is the most water soluble compound among the hits from our virtual screening.

240 The BOILED-Egg is a simple yet intuitive model for predicting small molecules' oral 241 bioavailability (Daina \& Zoete, 2016). When we plotted WLOGP and TPSA of the virtual

242 screening hits on the BOILED-Egg (Fig. 2), 11 ligands were inside the egg, the area representing 243 suitable physicochemical space for oral bioavailability. In the context of COVID-19 treatment, 244 candidate compounds in the egg white, which implies human intestinal absorption (HIA) without 245 blood-brain barrier (BBB) permeation, would be preferred for quicker drug development. Four 246 molecules inside the yellow are predicted to be distributed in the brain tissue. Nonetheless, these 247 four compounds seem to be P-glycoprotein (PGP) substrates, and thus, likely to be effluated from 248 the central nervous system. Although nine molecules are in the gray area, they are still close to 249 the egg's white and would gain better bioavailability profiles during a drug development phase.

250 Together, most of the hits from the MyriaScreen Diversity Library II virtual screening possess 251 optimum physicochemical characteristics for oral bioavailability.

252 Five major isoforms of cytochromes P450 (CYP1A2, CYP2C19, CYP2C9, CYP2D6, CYP3A4) 253 profoundly impact drug metabolism and elimination. Consequently, these isozymes are key 254 regulators of drug-drug interactions which in turn can dictate efficacy and adverse effects. Table 2553 provides data on whether top virtual screening hits can inhibit key CYP isozymes. We found 256 that molecules \#17 and \#20 are likely to exhibit greater drug-drug interactions as they would 257 inhibit four and five isozymes, respectively. On the other hand, \#9 and \#12 are inhibitors for none 258 of these metabolic enzymes. Table 3 also shows predicted plasma half-life $\left(\mathrm{T}_{1 / 2}\right)$ and clearance of 259 the short-listed molecules.

260 Lipinski’s rule of five (Lipinski et al., 2001) is extensively used in predicting druglikeness of 261 small molecules. A better plasma membrane permeability is assumed when a compound obeys 262 the following criteria: $\mathrm{MW} \leq 500, \mathrm{MLOGP} \leq, \mathrm{N}$ or $\mathrm{O} \leq 10$, and $\mathrm{NH}$ or $\mathrm{OH} \leq 5$. As expected, all of 263 the top 20 ligands followed the Lipinski's rule (Table 4). Most compounds also agreed with other 264 models of druglikeness, namely Ghose (Ghose, Viswanadhan \& Wendoloski, 1999), Veber 265 (Daniel F. Veber et al., 2002), Egan (Egan, Merz, \& Baldwin, 2000), and Muegge (Muegge, 266 Heald \& Brittelli, 2001).

267 We next computed toxicity profiles of the ligands using the ADMETlab webserver (Dong et al., 268 2018b), and OSIRIS Property Explorer (Sander, 2017). Table 5 demonstrates that nine of the top 269 ligands could show high toxicities. To note, \#1 molecule (R897698) is predicted to have medium 270 cardiac and mutagenic toxicities and high tumorigenicity. On the other hand, \#14 compound 271 (S51765) seems to be a safer lead without any major toxicity.

\section{Protein-Ligand Interaction Analysis}

273 When we considered AMDE/Tox profiles of the top 20 hits from the virtual screening, four 274 compounds stand out: L220477, R872172, ST074801, and S51765 (Fig. 3). These molecules 275 have physicochemical properties suitable for oral bioavailability, are predicted not to cross the 
$276 \mathrm{BBB}$, and seem to pose lower toxicity risks. Molecular docking confirmed that these four ligands 277 can occupy the active sites of the SARS-CoV-2 main protease (Fig. 3A). Figure 3B shows

278 multiple interactions of 3CL-PRO with the inhibitor 11a in 6LZE. L220477, R872172,

279 ST074801, and S51765 also interact with the critical residues of the protease. Radar charts depict 280 that lipophilicity, size, polarity, insolubility, insaturation, and flexibility of these compounds 281 favor gastrointestinal absorption ( $F i g .3 D, G, J, M$ ). Interestingly, S51765 resides entirely in the 282 physicochemical space for oral bioavailability (Fig. $3 L, M$ ). It is also tempting to note that this 283 molecule exhibits the least toxicity risks among the top 20 hits (Table 5).

\section{Molecular Docking with AutoDock4.2}

285 Although both AutoDock Vina and AutoDock4.2 are widely used for molecular docking and 286 outperform many docking tools in scoring performance, there is a speed-accuracy trade off 287 (Durrant et al., 2013; Wang et al., 2016; Gaillard, 2018; Nguyen et al., 2020). Compared to 288 Vina, AutoDock4.2 was found to generate superior binding affinity (Nguyen et al., 2020). We 289 performed flexible docking for the reference ligand 11a and the best four molecules from our 290 virtual screening. The most negative binding energy was obtained for $11 \mathrm{a}(-11.23 \mathrm{kcal} / \mathrm{mol})$ 291 followed by L220477 (-10.39 kcal/mol), R872172 (-10.26 kcal/mol), ST074801 (-10.17 $292 \mathrm{kcal} / \mathrm{mol})$, and S51765 (-10.06 kcal/mol). Computed inhibition constants were 5.85, 24.03, 30.11, 293 35.29, and 42.55 nM for 11a, L220477, R872172, ST074801, and S51765, respectively. These 294 295 results indicated the best four compounds from our virtual screening were almost identical in terms of AutoDock4.2 binding affinity.

\section{Validation of Protein-ligand Binding with Molecular Dynamics Simulations}

297

298

299

300

301

302

303

304

305

306

307

308

309

310

311

312

313

314

315
MD simulation studies have significant positive impacts on the drug discovery process (Ganesan, Coote \& Barakat, 2017; Liu et al., 2018; Guterres \& Im, 2020). We performed duplicated 50-ns MD simulations for AutoDock4.2-generated protein-ligand complexes to validate interactions of the candidate molecules with the SARS-CoV-2 main protease. As a reference, we included the crystal structure 6LZE, where the main protease is complexed with the ligand 11a. The solvent and ions of the simulation systems converged to a minimum energy level within 1500 minimization steps and subsequently attained NVT and NPT equilibria (Fig. S2). We analyzed the simulation trajectories to predict spatial fluctuations of the protein and ligands in complexes are summarized computations in Fig. 4 and Fig. S3, for the first and the second simulation, respectively. In the first simulation, mean RMSD values ( \pm SD) of the 3CL-PRO's C- $\alpha$ atoms were 2.19 ( \pm 0.64$), 1.92$ ( \pm 0.27$), 2.1$ ( \pm 0.36$), 2.92$ ( \pm 1$)$, and $1.69( \pm 0.23)$ angstroms for complexes with11a, L220477, R872172, ST074801, and S51765, respectively (Fig. 4A).

In the first simulation, the protein in the 3CL-PRO-11a complex showed initial fluctuations and the reference ligand 11a remained close to the binding pocket after an initial displacement (Fig. 4A, B). Although the protein was fairly stable with R872172 and L220477 (Fig 4A), Ligand RMSD values indicate wide fluctuations of the compounds (Fig. 4B). When complexed with ST074801, 3CL-PRO seemed to become very unstable at the end of the simulation and the ligand exhibited substantial fluctuations, indicating overall instability of the complex. On the other hand, 3CL-PRO-S51765 complex showed considerable stability (Fig. 4B). 
316 Mean ( \pm SD) RMSF values of alpha carbon atoms were $1.4( \pm 0.57), 1.13( \pm 0.57), 1.16( \pm 0.65)$, 3171.67 ( \pm 0.89), and 0.96 ( \pm 0.54) angstroms for 11a, L220477, R872172, ST074801, and S51765, 318 respectively (Fig. 4C). The RMSD of all four candidate molecules from the protein backbone 319 were very low, even lower than that of the reference ligand (Fig. 4B). We did not observe any 320 apparent differences in the ligand RMSF (Fig. 4D).

321 The reference ligand showed a higher RMSD in the second simulation. Compared to the first 322 simulation (Fig. 4B), S51765 also exhibited a higher RMSD value in the second simulation (Fig. $S 3 B$ ). When we repeated the simulation three more times, S51765 indicated considerable stability of the complex with low RMSD values (Fig. S4B). 3CL-PRO became unstable with R72172 and the ligand left the cavity (Fig. S3A, B). Interestingly, L220477 showed the least fluctuations (Fig. $S 3 B$ ). However, this ligand moved out of the binding pocket in repeated MD simulations (Fig. S4E, F). ST074801 also could not form a stable complex (Fig. S3B, Fig. S4C, D).

To have a closer look at the binding modes, we extracted frames at every 10 ns from the trajectories and rendered the ligands at the binding cavity (Fig. 5). Interestingly, the reference ligand 11a showed initial displacement at the binding cavity from 0 ns to 10 ns while maintaining contacts with HIS41 throughout the simulation. At around 40 ns, 11a seemed to momentarily move away from GLU166. L220477 (Fig. 5B1-B6) and R872172 (Fig. 5C1-C6) showed erratic fluctuations indicating unstable complex formation. The conformation of ST074801 in the binding cavity changed significantly during the first 10 ns of simulation (Fig. 5D1-D6). Intriguingly, the molecule S51765 settled very well in the cavity following a slight displacement at the beginning, (Fig. 5E1-E6). We further analyzed binding poses of S51765 in duplicate simulations (Fig. S5). In one case (simulation-2, Fig. S5A1-A6), the binding poses differed from other simulation. Nevertheless, S51765 showed consistency in most of the MD simulations, suggesting stability of the 3CL-PRO-S51765 complex.

We next analyzed the hydrogen bonds between 3CL-PRO and the selected ligands setting $3 \AA$ as the maximum donor-acceptor distance in VMD. Numbers of hydrogen bonds were plotted over the simulation period in Fig. 6A-E (first simulation) and Fig. S6A-E (second simulation). Occupancy of hydrogen bonds were shown in Table S2. We also plotted hydrogen bond occupancy by ligands (Fig. 6F, Fig. S6F) and by major amino acids in the binding pocket of 3CL-PRO (Fig. 6G, Fig. S6G). Clearly, the reference ligand 11a (Fig. 6A) exhibited the highest interactions over time, which was followed by S51765 (Fig. 6E) and ST074801 (Fig. 6D). Seemingly, L220477 and R872172 failed to establish sufficient hydrogen bonding for making stable complexes (Fig. 6B, C). Detail calculations identified residues HIS41, GLU166, and CYS145 as the best hydrogen bond donors for the reference ligand 11a in the crystal structure (Fig. 6G, Table S2). S51765 exhibited the highest occupancy for GLU166 followed by GLN189, MET165, and CYS145 whereas, ST074801 showed the highest interactions with GLN189 (Fig. 6G, Table S2).

We computed distances between the donor-acceptor atoms for the hydrogen bonds with the highest occupancy using the distance module of Gromacs (Table 6). The distance was below $3 \AA$ only in the 3CL-PRO-S51765 complex (Table 6). Intriguingly, the distance was highly consistent for this complex over the entire simulation period (Fig. 7E), whereas, the distance showed a high 
357 degree of fluctuation for other complexes (Fig. 7A-D). We also calculated the highest occupancy

358 protein-ligand hydrogen-bond distances for duplicate simulations of the 3CL-PRO-S51765

359 complex (Fig. S7). Except for simulation-2, the computed distances were highly indicative of a

360 stable complex formation.

361 To validate protein-ligand interactions further, we extracted two important energy terms from the

362 GROMACS MD simulation trajectories: short-range Coulomb (Coul-SR) and short-range

363 Lennard-Jones (LJ-SR) (Fig. 8). All ligands had negative values for both of the energies. Over

364 the 50-ns simulation period, the means ( \pm SD) of the sum of Coul-SR and LJ-SR were $-200( \pm$

365 28), -162 ( \pm 24$),-169$ ( \pm 24), -250 ( \pm 23$)$, and $-194( \pm 20) \mathrm{kJ} / \mathrm{mol}$ for 11a, L220477, R872172,

366 ST074801, and S51765, respectively (Fig. 8A). These results indicated that S51765 is capable of

367 forming a thermodynamically stable complex with the SARS-CoV-2 3CL-PRO.

368

369

370

371

372

373

374

375

376

377

378

379

380

381

382

383

384

385

386

387

388

389

390

391

392

393

394

395

\section{MMPBSA Binding Energy Calculation}

Binding free energy is a reliable measure of protein-ligand interactions. The Molecular Mechanics Poisson-Boltzmann Surface Area (MMPBSA) approach efficiently recapitulates the binding capacity of a small molecule to the target (Kumari et al., 2014; Wang et al., 2018). We computed the binding energy $(\mathrm{kJ} / \mathrm{mol})$ using the g_mmpbsa tool (Kumari et al., 2014) and results are presented in Table 7 . Total binding energy values were -72.95 and $-70.10 \mathrm{~kJ} / \mathrm{mol}$ for the reference ligand 11a and S51765, respectively. Compared to 11a, S51765 showed slightly higher van der Wall energy (170.17 kJ/mol vs. $159.31 \mathrm{~kJ} / \mathrm{mol})$ and slightly lower electrostatic energy ($23.35 \mathrm{~kJ} / \mathrm{mol}$ vs. $32.73 \mathrm{~kJ} / \mathrm{mol}$ ). There was no apparent difference in the polar solvation energy. Overall, the free energy signature of S51765 was almost identical with that of the reference ligand.

\section{Discussion}

To leave no stone unturned in discovering cures for COVID-19, the scientific community is deploying diverse approaches, from in silico to in vitro and from in vivo to clinical. We virtual screened the MyriaScreen Diversity Library II, an unexplored chemical space in the fight against the deadly SARS-CoV-2. This rich compound library from Sigma-Aldrich harbors 10,000 druglike entities encompassing diverse chemotypes (Hole et al., 2015; Njikan et al., 2018; Prado et al., 2018; Jain et al., 2020). A comprehensive in silico approach helped us identify at least four novel leads to design antiviral agents for treating COVID-19. Our computational study will accelerate future in vitro and in vivo experiments to discover antiviral agents for COVID-19.

Target-based virtual screening studies often rely on a single crystallographic structure. With the rapidly evolving COVID-19 situation, we see a surge in crystallographic studies of viral proteins. Now one has the luxury to choose from more than two hundred x-ray crystallographic structures available in the Protein Data Bank (www.rcsb.org) for the replicase polyprotein 1ab (also known as pp1ab) (UniProt accession code P0DTD1), the precursor of SARS-CoV-2 3CL-PRO. Inhibitor-bound crystal structures provide substantial insight into the protein's active sites to devise target-based inhibitors. Nevertheless, an X-ray crystallographic structure is a snapshot of a particular state, whereas the protein is very much dynamic and can adopt numerous forms in 
396

397

398

399

400

401

402

403

404

405

406

407

408

409

410

411

412

413

414

415

416

417

418

419

420

421

422

423

424

425

426

427

428

429

430

431

432

433

434

435 vivo. Conformational changes often occur from the unbound (also known as apo) to the substratebound (also known as holo) state. Moreover, a protein can undergo structural alterations depending on intra- and inter-molecular interactions.

Presumably, virtual screening of thousands of compounds using only a single target structure is very prone to miss potential ligands. Instead, we used 33 conformations of the 3CL-PRO from molecular dynamics simulations of three high-resolution crystallographic structures (PDB IDs 6LZE, 6M0K, and 6YB\&). We feel that this attempt was rewarded. Ten hits from the combined screening were absent in individual top lists for 6LZE and 6M0K (Table S3). Again, we would have missed 15 of the combined top-ranked molecules if we would consider only 6YB7. The ligand S51765 ranked 135, 88, and 22 when only 6LZE, 6M0K, and 6YB7, respectively, were used singly. Intriguingly, this very ligand turned out to one of the best potential leads in this study. Seemingly, employing many biologically relevant structures of the same target protein can enable capturing potential ligands that would otherwise remain unidentified.

Computational ADMET prediction can profoundly accelerate drug discovery programs by eliminating compounds with unfavorable physicochemical characteristics and toxicity profiles at an earlier stage. In our study, we used SwissADME (Daina, Michielin \& Zoete, 2017), ADMETlab (Dong et al., 2018b), and OSIRIS (Sander, 2017), which are some of the most advanced and widely used tools (Ferreira \& Andricopulo, 2019; Kar \& Leszczynski, 2020). However, validation of our ADMET prediction would be difficult as there is no recognized 3CLPRO inhibitors with known clinical data.

Intriguingly, the molecule S51765 is a macrocycle with 19 atoms in the ring. A recent study also identified a macrocyclic biomolecule (PubChem ID: 118098670) as a putative 3CL-PRO inhibitor through screening of protease inhibitors (Havranek \& Islam, 2020). Another macrocyclic protease inhibitor Danoprevir (DrugBank accession number: DB11779), an antiviral agent, was used in a clinical trial for COVID-19 (ClinicalTrials.gov Identifier: NCT04345276). Macrocycles present both an opportunity and a challenge for computational drug discovery. This group of compounds are emerging as promising leads which offer high bioavailability with enhanced affinity and selectivity for drug targets (Driggers et al., 2008; Mallinson \& Collins, 2012; Heinis, 2014). Although large cyclic compounds are generally difficult to model using docking tools, their active conformations could be obtained with higher confidence when molecular dynamics-based computation methods are employed (Sindhikara et al., 2017; Ugur et al., 2019).

Since the outbreak of the COVID-19 outbreak, many computational studies have been conducted to unveil potentials 3CL-PRO inhibitors from diverse sources including FDA-approved drugs, natural products, synthetic small molecules, and synthetic peptides. For example, in silico screening identified novel inhibitors from flavonoids (Gorla et al., 2020; Batool et al., 2020), marine products (Gentile et al., 2020), protease inhibitors (Havranek \& Islam, 2020; Keretsu, Bhujbal \& Cho, 2020), and commercial chemical libraries (Gimeno et al., 2020; Ibrahim et al., 2020; Uniyal et al., 2020). To our knowledge, no other study screened the MyriaScreen Diversity Library II for 3CL-PRO. 
436 Virtual screening through molecular docking has several limitations including variability in 437 predicted scores (Corbeil, Williams \& Labute, 2012; Koes, Baumgartner \& Camacho, 2013). To 438 circumvent the caveats partially, we adopted a number of measures. We attempted to minimize 439 false positives by comparing active-decoys, using multiple target structures, and repeating 440 molecular docking. We next enriched the top ligands by careful ADMET profiling. Finally, we 441 analyzed protein-ligand interactions through duplicated MD simulations and free energy 442 calculations. Conceivably, our in silico study could be an adjunct to, not a substitute for, 443 experimental validation of inhibitors for SARS-CoV-2 3CL-PRO.

\section{Conclusions}

445 The COVID-19 pandemic makes it imperative to find safe and effective remedies at the earliest 446 possible time. Computations studies can accelerate antiviral drug discovery by screening huge 447 small molecule libraries and providing leads for further development. In this study, we attempted 448 two goals, exploring a rich chemical library and maximizing the available structural information 449 of the target protein SARS-CoV-2 3CL-PRO. To mimic the dynamics in biological 450 environments, we generated many target conformations through MD simulations of three high451 resolution x-ray crystallographic structures of the viral protease. Subsequent virtual screening of 452 10,000 druglike small molecules in the MyriaScreen Diversity Library II unveils 20 candidate 453 ligands against a total of 33 conformations of 3CL-PRO. We identified four promising leads via 454 scrupulous physicochemical, biopharmaceutic, and toxicity profiling of top-ranked compounds 455 (Table 1-5). Visual inspection of protein-ligands interactions also suggested that those four 456 molecules could inhibit the SARS-CoV-2 main protease (Fig. 3).

457 We validated protein binding of the best four molecules by duplicated 50-ns MD simulations 458 (Fig. 4-8). Figure 5E1-E6 clearly shows that S51765 could form a stable complex since the 459 ligand was confined in the binding pocket of 3CL-PRO with only a subtle fluctuation during the 460 simulated period. Hydrogen bonding is the most ubiquitous non-bonded interactions in ligand 461 binding (Böhm \& Schneider, 2003; Williams \& Ladbury, 2005). Interestingly, S51765 exhibited 462 significant hydrogen bonding interactions (Fig. 7E) involving key residues for inhibitor binding 463 of 3CL-PRO (Zhang et al., 2020; Jin et al., 2020). This was also substantiated by favorable 464 interaction energies for S51765 (Fig. 8F, Table 7). Together, our comprehensive in silico studies 465 present S51765 a promising candidate molecule for developing 3CL-PRO inhibitors.

\section{Acknowledgments}

467 We are grateful to Dr. Muhammad Maqsud Hossain, Director, NSU Genome Research Institute 468 (NGRI), North South University, Bangladesh for allowing us remote access to the high469 performance computing facility of the NGRI. 


\section{References}

471

Abraham MJ, Murtola T, Schulz R, Páll S, Smith JC, Hess B, Lindahl E. 2015. GROMACS: High performance molecular simulations through multi-level parallelism from laptops to supercomputers. SoftwareX 1-2:19-25. DOI: 10.1016/j.softx.2015.06.001.

Anand K, Ziebuhr J, Wadhwani P, Mesters JR, Hilgenfeld R. 2003. Coronavirus Main Proteinase (3CLpro) Structure: Basis for Design of Anti-SARS Drugs. Science 300:1763-1767. DOI: 10.1126/science.1085658.

Arnott JA, Planey SL. 2012. The influence of lipophilicity in drug discovery and design. Expert Opinion on Drug Discovery 7:863-875. DOI: 10.1517/17460441.2012.714363.

Batool F, Mughal EU, Zia K, Sadiq A, Naeem N, Javid A, Ul-Haq Z, Saeed M. 2020. Synthetic flavonoids as potential antiviral agents against SARS-CoV-2 main protease. Journal of Biomolecular Structure and Dynamics 0:1-12. DOI: 10.1080/07391102.2020.1850359.

Berendsen HJC, van der Spoel D, van Drunen R. 1995. GROMACS: A message-passing parallel molecular dynamics implementation. Computer Physics Communications 91:4356. DOI: 10.1016/0010-4655(95)00042-E.

BIOVIA, Dassault Systèmes, Discovery Studio Visualizer, v20.1.0.192. 2019. San Diego: Dassault Systèmes.

Böhm H $\square$ J., Schneider G (eds.). 2003. Protein Ligand Interactions: From Molecular Recognition to Drug Design. Wiley. DOI: 10.1002/3527601813.

CGenFF Home. Available at https://cgenff.umaryland.edu/ (accessed November 2, 2020).

Corbeil CR, Williams Cl, Labute P. 2012. Variability in docking success rates due to dataset preparation. Journal of Computer-Aided Molecular Design 26:775-786. DOI: 10.1007/s10822-012-9570-1.

Cui W, Aouidate A, Wang S, Yu Q, Li Y, Yuan S. 2020. Discovering Anti-Cancer Drugs via Computational Methods. Frontiers in Pharmacology 11. DOI: 10.3389/fphar.2020.00733. 
Dai W, Zhang B, Jiang X-M, Su H, Li J, Zhao Y, Xie X, Jin Z, Peng J, Liu F, Li C, Li Y, Bai F, Wang H, Cheng X, Cen X, Hu S, Yang X, Wang J, Liu X, Xiao G, Jiang H, Rao Z, Zhang L-K, Xu Y, Yang H, Liu H. 2020. Structure-based design of antiviral drug candidates targeting the SARS-CoV-2 main protease. Science 368:1331-1335. DOI: 10.1126/science.abb4489.

Daina A, Michielin O, Zoete V. 2017. SwissADME: a free web tool to evaluate pharmacokinetics, drug-likeness and medicinal chemistry friendliness of small molecules. Scientific Reports 7:42717. DOI: 10.1038/srep42717.

Daina A, Zoete V. 2016. A BOILED $\square$ Egg To Predict Gastrointestinal Absorption and Brain Penetration of Small Molecules. Chemmedchem 11:1117-1121. DOI: 10.1002/cmdc.201600182.

Daniel F. Veber *, Stephen R. Johnson ¥, Hung-Yuan Cheng II, Brian R. Smith $\perp$, Keith W. Ward $\perp$ and, Kenneth D. Kopple \#. 2002. Molecular Properties That Influence the Oral Bioavailability of Drug Candidates. Available at https://pubs.acs.org/doi/pdfplus/10.1021/jm020017n (accessed October 5, 2020). DOI: 10.1021/jm020017n.

Delaney JS. 2004. ESOL: Estimating Aqueous Solubility Directly from Molecular Structure. Journal of Chemical Information and Computer Sciences 44:1000-1005. DOI: 10.1021/ci034243x.

Dong R, Peng Z, Zhang Y, Yang J. 2018a. mTM-align: an algorithm for fast and accurate multiple protein structure alignment. Bioinformatics 34:1719-1725. DOI: 10.1093/bioinformatics/btx828.

Dong J, Wang N-N, Yao Z-J, Zhang L, Cheng Y, Ouyang D, Lu A-P, Cao D-S. 2018b. ADMETlab: a platform for systematic ADMET evaluation based on a comprehensively collected ADMET database. Journal of Cheminformatics 10:29. DOI: 10.1186/s13321018-0283-x. 
Driggers EM, Hale SP, Lee J, Terrett NK. 2008. The exploration of macrocycles for drug discovery — an underexploited structural class. Nature Reviews Drug Discovery 7:608624. DOI: $10.1038 / \mathrm{nrd} 2590$.

Durrant JD, Friedman AJ, Rogers KE, McCammon JA. 2013. Comparing Neural-Network Scoring Functions and the State of the Art: Applications to Common Library Screening. Journal of Chemical Information and Modeling 53:1726-1735. DOI: 10.1021/ci400042y.

Egan WJ, Merz, KM, Baldwin JJ. 2000. Prediction of Drug Absorption Using Multivariate Statistics. Journal of Medicinal Chemistry 43:3867-3877. DOI: 10.1021/jm000292e.

Ferreira LLG, Andricopulo AD. 2019. ADMET modeling approaches in drug discovery. Drug Discovery Today 24:1157-1165. DOI: 10.1016/j.drudis.2019.03.015.

Forster PM, Forster HI, Evans MJ, Gidden MJ, Jones CD, Keller CA, Lamboll RD, Quéré CL, Rogelj J, Rosen D, Schleussner C-F, Richardson TB, Smith CJ, Turnock ST. 2020. Current and future global climate impacts resulting from COVID-19. Nature Climate Change 10:913-919. DOI: 10.1038/s41558-020-0883-0.

Gaillard T. 2018. Evaluation of AutoDock and AutoDock Vina on the CASF-2013 Benchmark. J. Chem. Inf. Model.:10.

Ganesan A, Coote ML, Barakat K. 2017. Molecular dynamics-driven drug discovery: leaping forward with confidence. Drug Discovery Today 22:249-269. DOI:

10.1016/j.drudis.2016.11.001.

Gentile D, Patamia V, Scala A, Sciortino MT, Piperno A, Rescifina A. 2020. Putative Inhibitors of SARS-CoV-2 Main Protease from A Library of Marine Natural Products: A Virtual Screening and Molecular Modeling Study. Marine Drugs 18:225. DOI: 10.3390/md18040225.

Ghose AK, Viswanadhan VN, Wendoloski JJ. 1999. A knowledge-based approach in designing combinatorial or medicinal chemistry libraries for drug discovery. 1. A qualitative and quantitative characterization of known drug databases. Journal of Combinatorial Chemistry 1:55-68. DOI: 10.1021/cc9800071. 
Gimeno A, Mestres-Truyol J, Ojeda-Montes MJ, Macip G, Saldivar-Espinoza B, CeretoMassagué A, Pujadas G, Garcia-Vallvé S. 2020. Prediction of Novel Inhibitors of the Main Protease (M-pro) of SARS-CoV-2 through Consensus Docking and Drug Reposition. International Journal of Molecular Sciences 21:3793. DOI: 10.3390/ijms21113793.

Gorbalenya AE, Baker SC, Baric RS, de Groot RJ, Drosten C, Gulyaeva AA, Haagmans BL, Lauber C, Leontovich AM, Neuman BW, Penzar D, Perlman S, Poon LLM, Samborskiy DV, Sidorov IA, Sola I, Ziebuhr J, Coronaviridae Study Group of the International Committee on Taxonomy of Viruses. 2020. The species Severe acute respiratory syndrome-related coronavirus : classifying 2019-nCoV and naming it SARS-CoV-2. Nature Microbiology 5:536-544. DOI: 10.1038/s41564-020-0695-z.

Gordon DE, Jang GM, Bouhaddou M, Xu J, Obernier K, White KM, O'Meara MJ, Rezelj VV, Guo JZ, Swaney DL, Tummino TA, Hüttenhain R, Kaake RM, Richards AL, Tutuncuoglu B, Foussard H, Batra J, Haas K, Modak M, Kim M, Haas P, Polacco BJ, Braberg H, Fabius JM, Eckhardt M, Soucheray M, Bennett MJ, Cakir M, McGregor MJ, Li Q, Meyer B, Roesch F, Vallet T, Mac Kain A, Miorin L, Moreno E, Naing ZZC, Zhou Y, Peng S, Shi Y, Zhang Z, Shen W, Kirby IT, Melnyk JE, Chorba JS, Lou K, Dai SA, Barrio-Hernandez I, Memon D, Hernandez-Armenta C, Lyu J, Mathy CJP, Perica T, Pilla KB, Ganesan SJ, Saltzberg DJ, Rakesh R, Liu X, Rosenthal SB, Calviello L, Venkataramanan S, LiboyLugo J, Lin Y, Huang X-P, Liu Y, Wankowicz SA, Bohn M, Safari M, Ugur FS, Koh C, Savar NS, Tran QD, Shengjuler D, Fletcher SJ, O’Neal MC, Cai Y, Chang JCJ, Broadhurst DJ, Klippsten S, Sharp PP, Wenzell NA, Kuzuoglu-Ozturk D, Wang H-Y, Trenker R, Young JM, Cavero DA, Hiatt J, Roth TL, Rathore U, Subramanian A, Noack J, Hubert M, Stroud RM, Frankel AD, Rosenberg OS, Verba KA, Agard DA, Ott M, Emerman M, Jura N, von Zastrow M, Verdin E, Ashworth A, Schwartz O, d'Enfert C, Mukherjee S, Jacobson M, Malik HS, Fujimori DG, Ideker T, Craik CS, Floor SN, Fraser JS, Gross JD, Sali A, Roth BL, Ruggero D, Taunton J, Kortemme T, Beltrao P, Vignuzzi M, García-Sastre A, Shokat KM, Shoichet BK, Krogan NJ. 2020. A SARS-CoV-2 protein 
interaction map reveals targets for drug repurposing. Nature 583:459-468. DOI: 10.1038/ s41586-020-2286-9.

Gorla US, Rao GK, Kulandaivelu US, Alavala RR, Panda SP. 2020. Lead Finding from Selected Flavonoids with Antiviral (SARS-CoV-2) Potentials against COVID-19: An in-silico Evaluation. Combinatorial Chemistry \& High Throughput Screening. DOI: $10.2174 / 1386207323999200818162706$.

Guterres H, Im W. 2020. Improving Protein-Ligand Docking Results with High-Throughput Molecular Dynamics Simulations. J. Chem. Inf. Model.:10.

Hartley DM, Perencevich EN. 2020. Public Health Interventions for COVID-19: Emerging Evidence and Implications for an Evolving Public Health Crisis. JAMA 323:1908. DOI: 10.1001/jama.2020.5910.

Havranek B, Islam SM. 2020. An in silico approach for identification of novel inhibitors as potential therapeutics targeting COVID-19 main protease. Journal of Biomolecular Structure \& Dynamics:1-12. DOI: 10.1080/07391102.2020.1776158.

Headey D, Heidkamp R, Osendarp S, Ruel M, Scott N, Black R, Shekar M, Bouis H, Flory A, Haddad L, Walker N. 2020. Impacts of COVID-19 on childhood malnutrition and nutritionrelated mortality. The Lancet 396:519-521. DOI: 10.1016/S0140-6736(20)31647-0.

Heinis C. 2014. Tools and rules for macrocycles. Nature Chemical Biology 10:696-698. DOI: 10.1038/nchembio.1605.

Hole M, Underhaug J, Diez H, Ying M, Røhr ÅK, Jorge-Finnigan A, Fernàndez-Castillo N, García-Cazorla A, Andersson KK, Teigen K, Martinez A. 2015. Discovery of compounds that protect tyrosine hydroxylase activity through different mechanisms. Biochimica et Biophysica Acta (BBA) - Proteins and Proteomics 1854:1078-1089. DOI: 10.1016/j.bbapap.2015.04.030.

Huang C, Wang Y, Li X, Ren L, Zhao J, Hu Y, Zhang L, Fan G, Xu J, Gu X, Cheng Z, Yu T, Xia J, Wei Y, Wu W, Xie X, Yin W, Li H, Liu M, Xiao Y, Gao H, Guo L, Xie J, Wang G, Jiang R, Gao Z, Jin Q, Wang J, Cao B. 2020. Clinical features of patients infected with 2019 
novel coronavirus in Wuhan, China. The Lancet 395:497-506. DOI: 10.1016/S01406736(20)30183-5.

Humphrey W, Dalke A, Schulten K. 1996. VMD - Visual Molecular Dynamics. Journal of Molecular Graphics 14:33-38.

Ibrahim MAA, Abdeljawaad KAA, Abdelrahman AHM, Hegazy M-EF. 2020. Natural-like products as potential SARS-CoV-2 Mpro inhibitors: in-silico drug discovery. Journal of Biomolecular Structure \& Dynamics:1-13. DOI: 10.1080/07391102.2020.1790037.

Jain R, Gupta S, Munde M, Pati S, Singh S. 2020. Development of novel anti-malarial from structurally diverse library of molecules, targeting plant-like CDPK1, a multistage growth regulator of P. falciparum. Biochemical Journal 477:1951-1970. DOI: 10.1042/BCJ20200045.

Jiang H, Li Y, Zhang H, Wang W, Yang X, Qi H, Li H, Men D, Zhou J, Tao S. 2020. SARS-CoV-2 proteome microarray for global profiling of COVID-19 specific IgG and IgM responses. Nature Communications 11:3581. DOI: 10.1038/s41467-020-17488-8.

Jin Z, Du X, Xu Y, Deng Y, Liu M, Zhao Y, Zhang B, Li X, Zhang L, Peng C, Duan Y, Yu J, Wang L, Yang K, Liu F, Jiang R, Yang X, You T, Liu X, Yang X, Bai F, Liu H, Liu X, Guddat LW, Xu W, Xiao G, Qin C, Shi Z, Jiang H, Rao Z, Yang H. 2020. Structure of M pro from SARS-CoV-2 and discovery of its inhibitors. Nature 582:289-293. DOI: 10.1038/s41586020-2223-y.

Joshi T, Sharma P, Joshi T, Pundir H, Mathpal S, Chandra S. 2020. Structure-based screening of novel lichen compounds against SARS Coronavirus main protease (Mpro) as potentials inhibitors of COVID-19. Molecular Diversity. DOI: 10.1007/s11030-020-10118$\mathrm{X}$.

Kapetanovic IM. 2008. Computer-aided drug discovery and development (CADDD): In silicochemico-biological approach. Chemico-Biological Interactions 171:165-176. DOI: 10.1016/j.cbi.2006.12.006. 
Kar S, Leszczynski J. 2020. Open access in silico tools to predict the ADMET profiling of drug candidates. Expert Opinion on Drug Discovery 15:1473-1487. DOI: 10.1080/17460441.2020.1798926.

Keretsu S, Bhujbal SP, Cho SJ. 2020. Rational approach toward COVID-19 main protease inhibitors via molecular docking, molecular dynamics simulation and free energy calculation. Scientific Reports 10:17716. DOI: 10.1038/s41598-020-74468-0.

Khanna V, Ranganathan S. 2009. Physiochemical property space distribution among human metabolites, drugs and toxins. BMC Bioinformatics 10:S10. DOI: 10.1186/1471-2105-10S15-S10.

Kim D, Lee J-Y, Yang J-S, Kim JW, Kim VN, Chang H. 2020. The Architecture of SARS-CoV-2 Transcriptome. Cell 181:914-921.e10. DOI: 10.1016/j.cell.2020.04.011.

Koes DR, Baumgartner MP, Camacho CJ. 2013. Lessons Learned in Empirical Scoring with smina from the CSAR 2011 Benchmarking Exercise. Journal of Chemical Information and Modeling 53:1893-1904. DOI: 10.1021/ci300604z.

Kumari R, Kumar R, Open Source Drug Discovery Consortium, Lynn A. 2014. g_mmpbsa—A GROMACS Tool for High-Throughput MM-PBSA Calculations. Journal of Chemical Information and Modeling 54:1951-1962. DOI: 10.1021/ci500020m.

Lindahl, Abraham, Hess, van der Spoel. 2019. GROMACS 2019.3 Source code. Zenodo. DOI: 10.5281/zenodo.3243833.

Lipinski CA, Lombardo F, Dominy BW, Feeney PJ. 2001. Experimental and computational approaches to estimate solubility and permeability in drug discovery and development settings1PII of original article: S0169-409X(96)00423-1. The article was originally published in Advanced Drug Delivery Reviews 23 (1997) 3-25.1. Advanced Drug Delivery Reviews 46:3-26. DOI: 10.1016/S0169-409X(00)00129-0.

Liu X, Shi D, Zhou S, Liu H, Liu H, Yao X. 2018. Molecular dynamics simulations and novel drug discovery. Expert Opinion on Drug Discovery 13:23-37. DOI:

10.1080/17460441.2018.1403419. 
Macalino SJY, Gosu V, Hong S, Choi S. 2015. Role of computer-aided drug design in modern drug discovery. Archives of Pharmacal Research 38:1686-1701. DOI: 10.1007/s12272015-0640-5.

Mallinson J, Collins I. 2012. Macrocycles in new drug discovery. Future Medicinal Chemistry 4:1409-1438. DOI: 10.4155/fmc.12.93.

McKibbin WJ, Fernando R. 2020. The Global Macroeconomic Impacts of COVID-19: Seven Scenarios. Rochester, NY: Social Science Research Network. DOI: 10.2139/ssrn.3547729.

Morris GM, Huey R, Lindstrom W, Sanner MF, Belew RK, Goodsell DS, Olson AJ. 2009. AutoDock4 and AutoDockTools4: Automated Docking with Selective Receptor Flexibility. Journal of computational chemistry 30:2785-2791. DOI: 10.1002/jcc.21256.

Muegge I, Heald SL, Brittelli D. 2001. Simple Selection Criteria for Drug-like Chemical Matter. Journal of Medicinal Chemistry 44:1841-1846. DOI: 10.1021/jm015507e.

Mysinger MM, Carchia M, Irwin JohnJ, Shoichet BK. 2012. Directory of Useful Decoys, Enhanced (DUD-E): Better Ligands and Decoys for Better Benchmarking. Journal of Medicinal Chemistry 55:6582-6594. DOI: 10.1021/jm300687e.

Nguyen NT, Nguyen TH, Pham TNH, Huy NT, Bay MV, Pham MQ, Nam PC, Vu VV, Ngo ST. 2020. Autodock Vina Adopts More Accurate Binding Poses but Autodock4 Forms Better Binding Affinity. Journal of Chemical Information and Modeling 60:204-211. DOI: 10.1021/acs.jcim.9b00778.

Njikan S, Manning AJ, Ovechkina Y, Awasthi D, Parish T. 2018. High content, high-throughput screening for small molecule inducers of NF-kB translocation. PLOS ONE 13:e0199966. DOI: 10.1371/journal.pone.0199966.

O’Boyle NM, Banck M, James CA, Morley C, Vandermeersch T, Hutchison GR. 2011. Open Babel: An open chemical toolbox. Journal of Cheminformatics 3:33. DOI: 10.1186/17582946-3-33. 
Pettersen EF, Goddard TD, Huang CC, Couch GS, Greenblatt DM, Meng EC, Ferrin TE. 2004. UCSF Chimera--a visualization system for exploratory research and analysis. Journal of Computational Chemistry 25:1605-1612. DOI: 10.1002/jcc.20084.

Prado S, Beltrán M, Moreno Á, Bedoya LM, Alcamí J, Gallego J. 2018. A small-molecule inhibitor of HIV-1 Rev function detected by a diversity screen based on RRE-Rev interference. Biochemical Pharmacology 156:68-77. DOI: 10.1016/j.bcp.2018.07.040.

Samdani A, Vetrivel U. 2018. POAP: A GNU parallel based multithreaded pipeline of open babel and AutoDock suite for boosted high throughput virtual screening. Computational Biology and Chemistry 74:39-48. DOI: 10.1016/j.compbiolchem.2018.02.012.

Sander T. 2017. OSIRIS Property Explorer.

Screening Compounds: MyriaScreen Diversity Collection. 2020. Available at https://www.sigmaaldrich.com/chemistry/chemistry-services/high-throughput-screening/ screening-compounds.html (accessed August 30, 2020).

Selvaraj C, Dinesh DC, Panwar U, Abhirami R, Boura E, Singh SK. 2020. Structure-based virtual screening and molecular dynamics simulation of SARS-CoV-2 Guanine-N7 methyltransferase (nsp14) for identifying antiviral inhibitors against COVID-19. Journal of Biomolecular Structure \& Dynamics:1-12. DOI: 10.1080/07391102.2020.1778535.

Sindhikara D, Spronk SA, Day T, Borrelli K, Cheney DL, Posy SL. 2017. Improving Accuracy, Diversity, and Speed with Prime Macrocycle Conformational Sampling. Journal of Chemical Information and Modeling 57:1881-1894. DOI: 10.1021/acs.jcim.7b00052.

Sultana F, Mahmud Reza H, 1 Department of Political Science and Sociology, North South University, Bashundhara R/A, Dhaka 1229, Bangladesh, 2 Department of Pharmaceutical Sciences, North South University, Bashundhara R/A, Dhaka 1229, Bangladesh. 2020. Are SAARC countries prepared to combat COVID-19 to save young, working-age population? AIMS Public Health 7:440-449. DOI:

10.3934/publichealth.2020036. 
Trott O, Olson AJ. 2010. AutoDock Vina: Improving the speed and accuracy of docking with a new scoring function, efficient optimization, and multithreading. Journal of Computational Chemistry 31:455-461. DOI: 10.1002/jcc.21334.

Ugur I, Schroft M, Marion A, Glaser M, Antes I. 2019. Predicting the bioactive conformations of macrocycles: a molecular dynamics-based docking procedure with DynaDock. Journal of Molecular Modeling 25:197. DOI: 10.1007/s00894-019-4077-5.

Uniyal A, Mahapatra MK, Tiwari V, Sandhir R, Kumar R. 2020. Targeting SARS-CoV-2 main protease: structure based virtual screening, in silico ADMET studies and molecular dynamics simulation for identification of potential inhibitors. Journal of Biomolecular Structure and Dynamics 0:1-17. DOI: 10.1080/07391102.2020.1848636.

Vanommeslaeghe K, Hatcher E, Acharya C, Kundu S, Zhong S, Shim J, Darian E, Guvench O, Lopes P, Vorobyov I, Mackerell AD. 2010. CHARMM general force field: A force field for drug-like molecules compatible with the CHARMM all-atom additive biological force fields. Journal of Computational Chemistry 31:671-690. DOI: 10.1002/jcc.21367.

Wang C, Greene D, Xiao L, Qi R, Luo R. 2018. Recent Developments and Applications of the MMPBSA Method. Frontiers in Molecular Biosciences 4:87. DOI:

10.3389/fmolb.2017.00087.

Wang Z, Sun H, Yao X, Li D, Xu L, Li Y, Tian S, Hou T. 2016. Comprehensive evaluation of ten docking programs on a diverse set of protein-ligand complexes: the prediction accuracy of sampling power and scoring power. Physical Chemistry Chemical Physics 18:1296412975. DOI: 10.1039/C6CP01555G.

Wildman SA, Crippen GM. 1999. Prediction of Physicochemical Parameters by Atomic Contributions. Journal of Chemical Information and Computer Sciences 39:868-873. DOI: $10.1021 /$ ci990307I.

Williams MA, Ladbury JE. 2005. Hydrogen Bonds in Protein-Ligand Complexes. In: ProteinLigand Interactions. John Wiley \& Sons, Ltd, 137-161. DOI: 10.1002/3527601813.ch6. Wu A, Peng Y, Huang B, Ding X, Wang X, Niu P, Meng J, Zhu Z, Zhang Z, Wang J, Sheng J, Quan L, Xia Z, Tan W, Cheng G, Jiang T. 2020. Genome Composition and Divergence of 
the Novel Coronavirus (2019-nCoV) Originating in China. Cell Host \& Microbe 27:325328. DOI: 10.1016/j.chom.2020.02.001.

Yu W, MacKerell AD. 2017. Computer-Aided Drug Design Methods. Methods in Molecular Biology (Clifton, N.J.) 1520:85-106. DOI: 10.1007/978-1-4939-6634-9_5.

Zhang L, Lin D, Sun X, Curth U, Drosten C, Sauerhering L, Becker S, Rox K, Hilgenfeld R. 2020. Crystal structure of SARS-CoV-2 main protease provides a basis for design of improved a-ketoamide inhibitors. Science 368:409-412. DOI: 10.1126/science.abb3405.

Zhou P, Yang X-L, Wang X-G, Hu B, Zhang L, Zhang W, Si H-R, Zhu Y, Li B, Huang C-L, Chen H-D, Chen J, Luo Y, Guo H, Jiang R-D, Liu M-Q, Chen Y, Shen X-R, Wang X, Zheng XS, Zhao K, Chen Q-J, Deng F, Liu L-L, Yan B, Zhan F-X, Wang Y-Y, Xiao G-F, Shi Z-L. 2020. A pneumonia outbreak associated with a new coronavirus of probable bat origin. Nature 579:270-273. DOI: 10.1038/s41586-020-2012-7.

Ziebuhr J, Snijder EJ, Gorbalenya AE. 2000. Virus-encoded proteinases and proteolytic processing in the Nidovirales. Journal of General Virology, 81:853-879. DOI: 10.1099/0022-1317-81-4-853. 


\section{Figure 1}

Molecular dynamics simulation of 3CL-PRO's three crystal structures (6LZE, 6M0K, and 6YB7).

(A) Alignment of three crystal structures. (B) Energy minimization for molecular dynamics simulation. (C) NVT equilibration. (D) NPT equilibration. (E-J) Conformational changes of four amino acid residues at the active site of 3CL-PRO over the simulation period. (K) RMSD (running averages) of alpha carbons. (L) RMSF of alpha carbons. Inset shows fluctuations of a loop region of $6 \mathrm{LZE}$. 

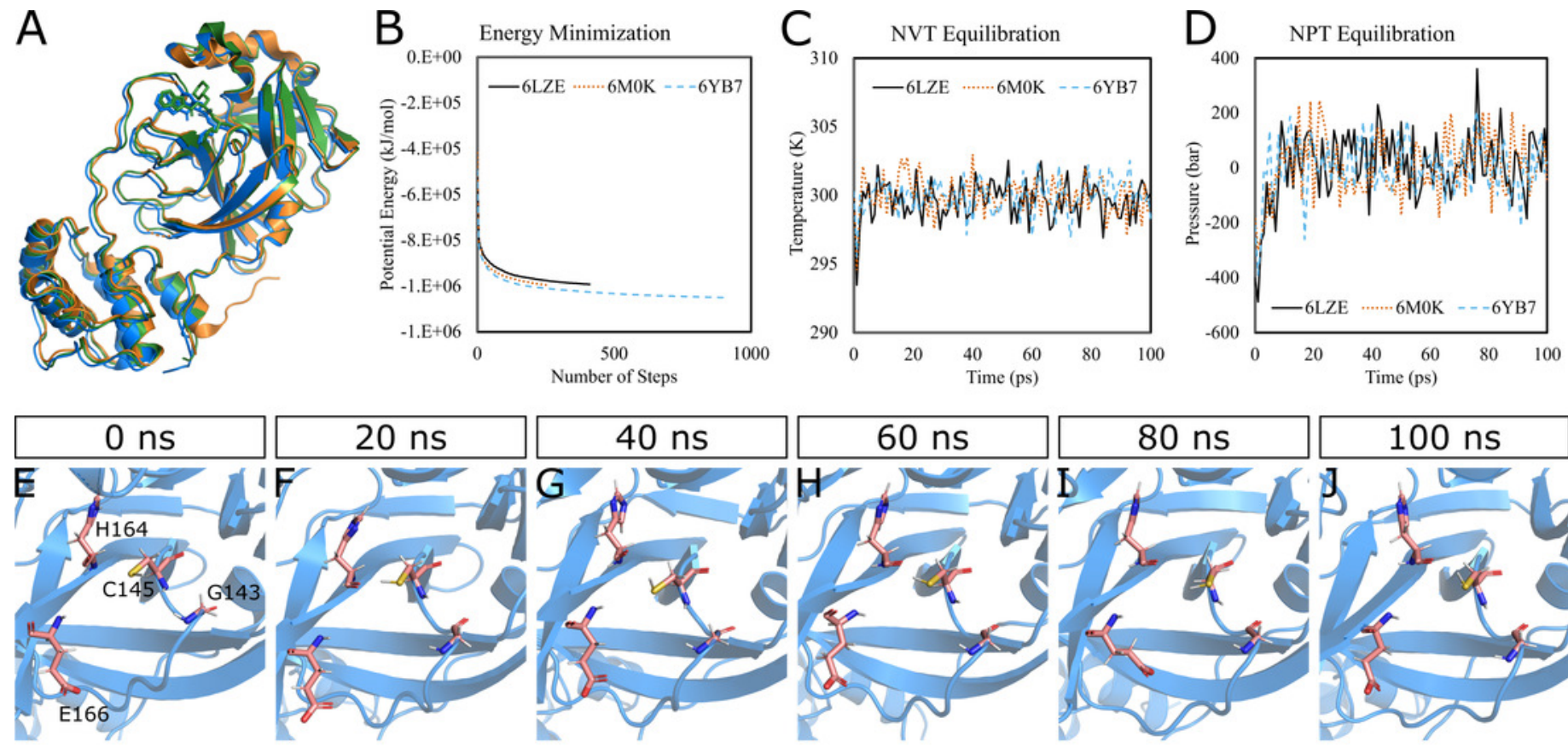

K

C-aplha RMSD
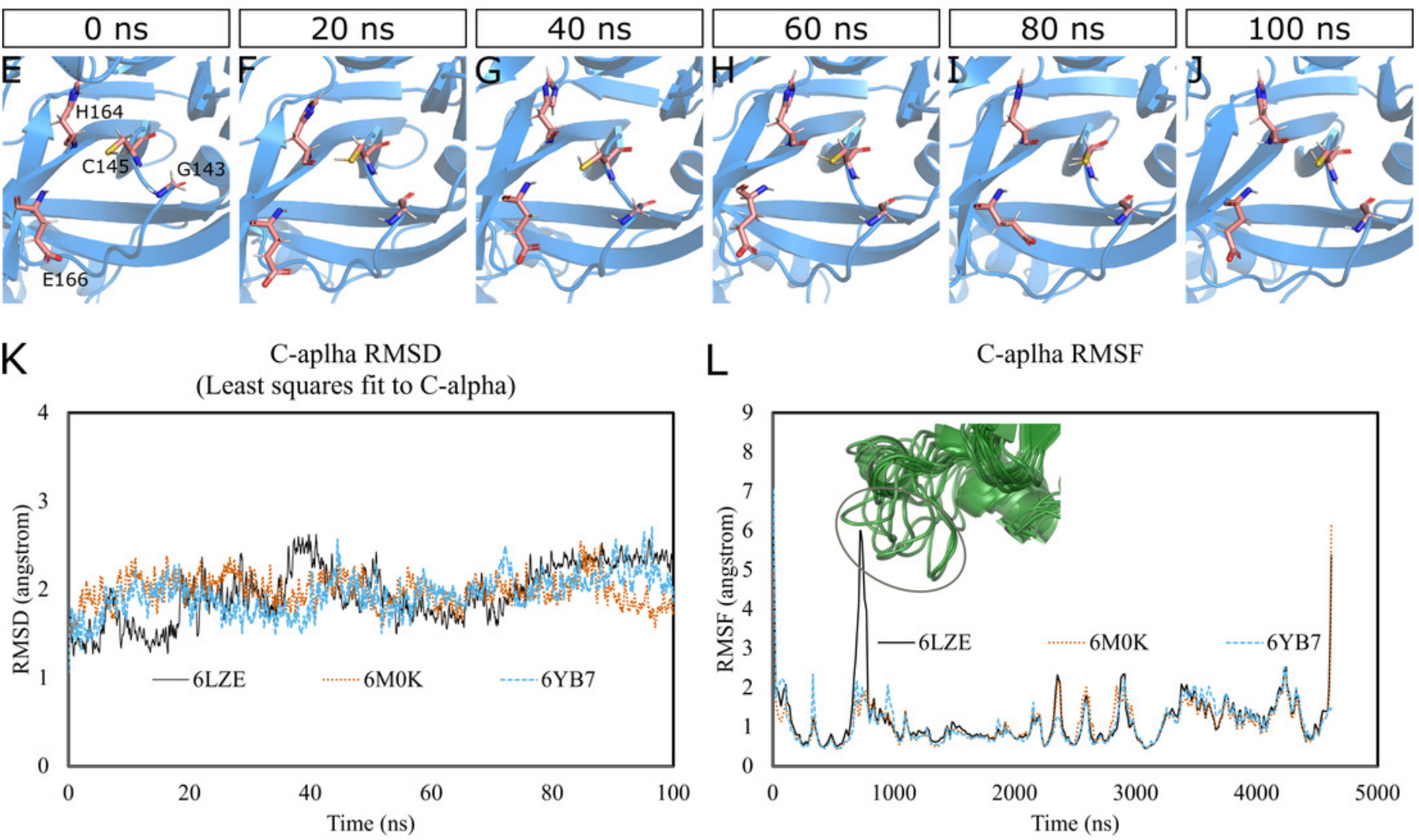

L

C-aplha RMSF

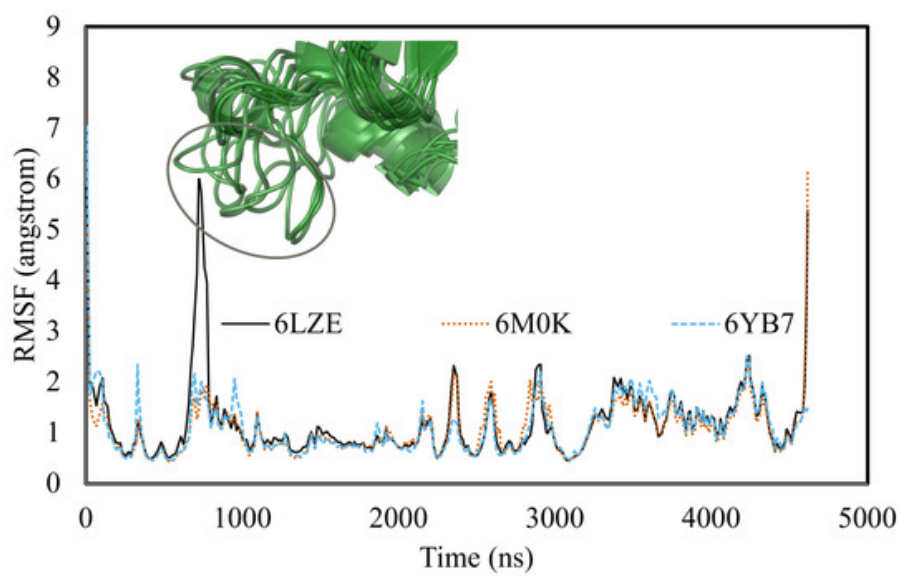


Figure 2

TPSA and WLOGP of top 20 ligands plotted on the BOILED-Egg.

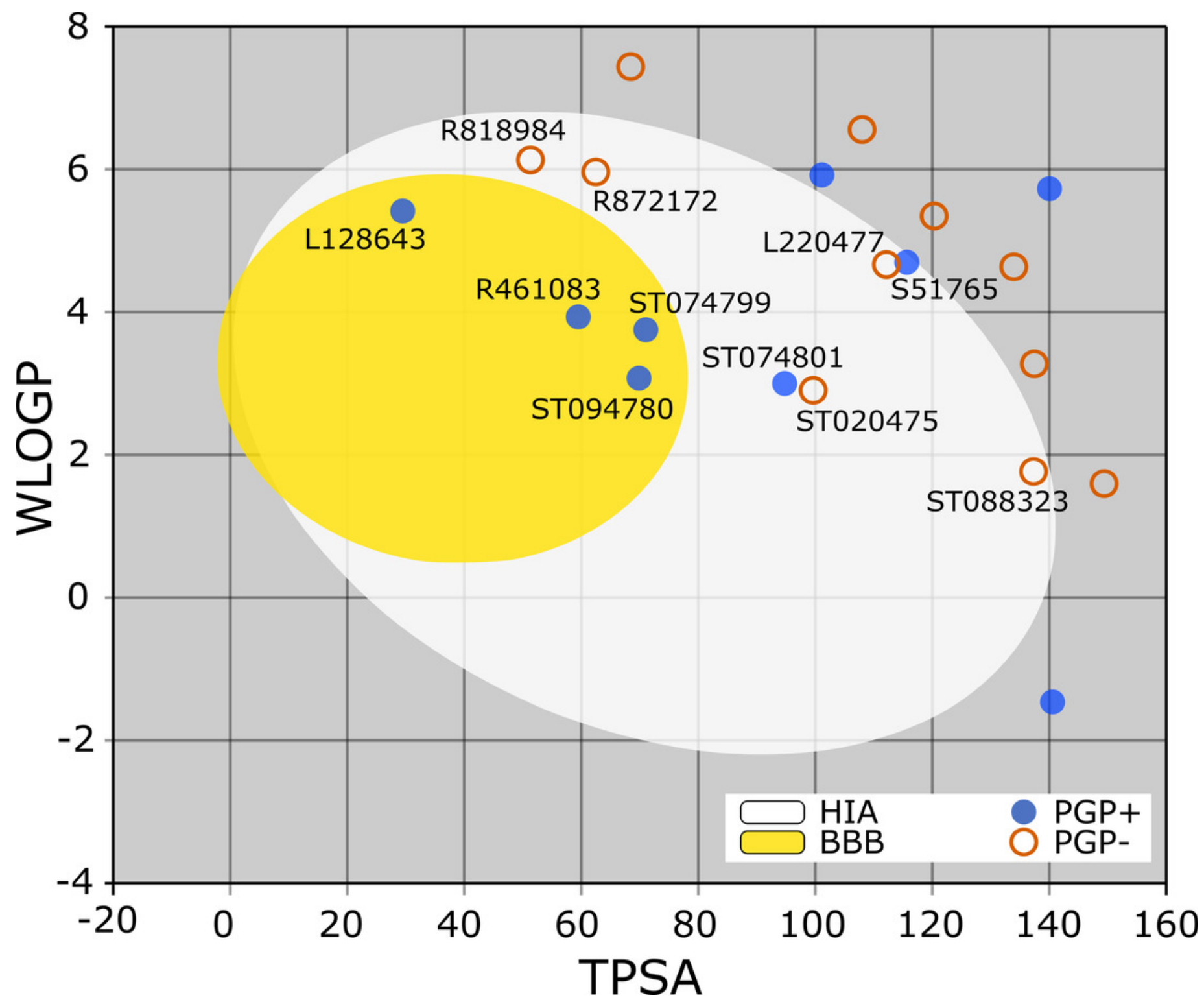




\section{Figure 3}

Docking conformations, physicochemical properties, and protein-ligand interactions for the best four molecules.

(A) Best docking poses of the ligands from virtual screening. In 6LZE, 11a is the cocrystallized ligand. (B) Interactions of 3CL-PRO and the ligand 11a in 6LZE. (C-N) Structure, physicochemical properties, and protein-ligand interactions of L220477 (C-D), R872172 (F-H), L220477 (I-K), and S51765 (L-N). The colored zone in radar charts (D, G, J, and M) indicates suitable physicochemical space for oral bioavailability. LIPO - lipophilicity (XLOGP3), SIZE molecular weight ( $\mathrm{g} / \mathrm{mol})$, POLAR - polarity (TPSA), INSOLU - insolubility (LogS), INSATU insaturation (fraction Csp3), FLEX - flexibility (number of rotatable bonds). 

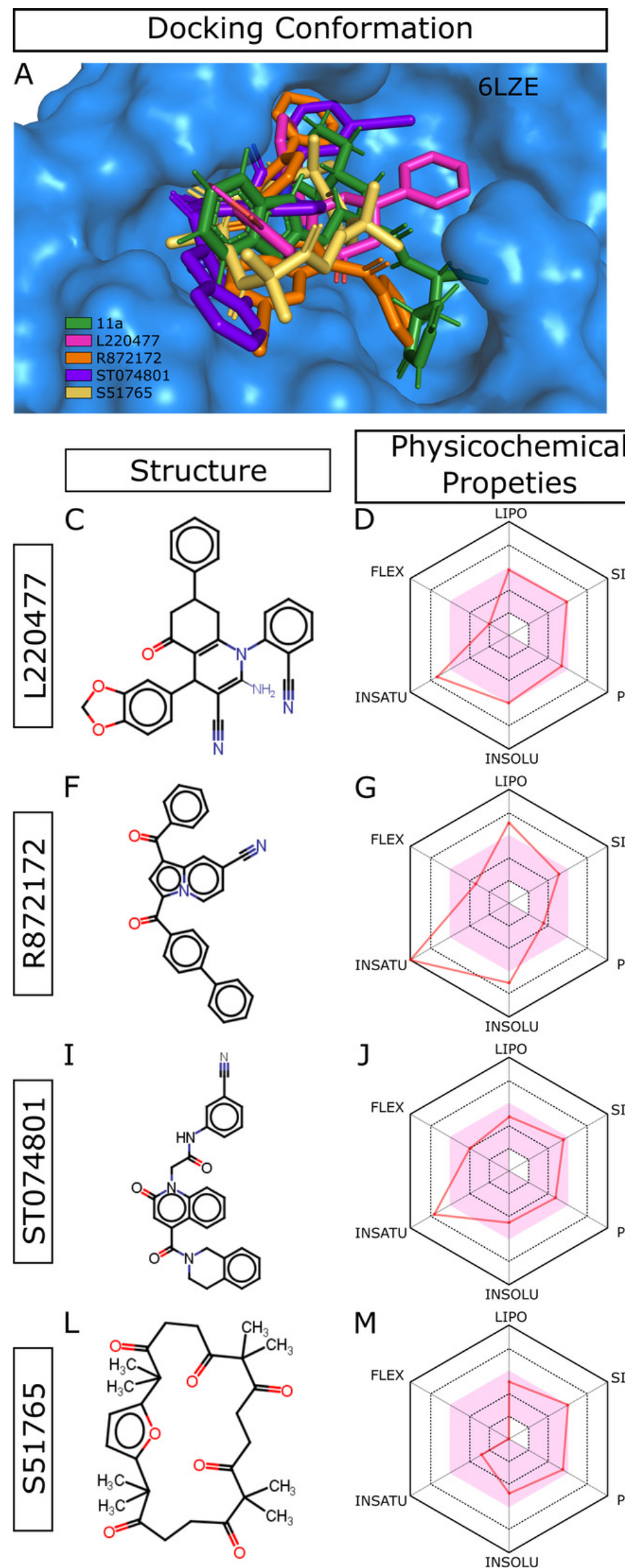

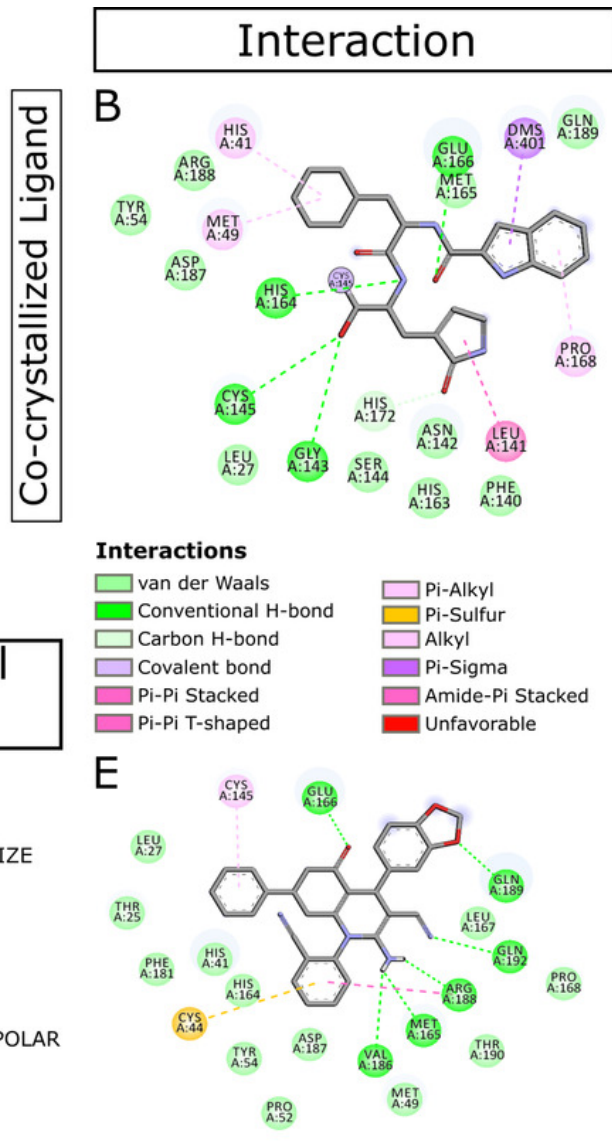

$\mathrm{H}$
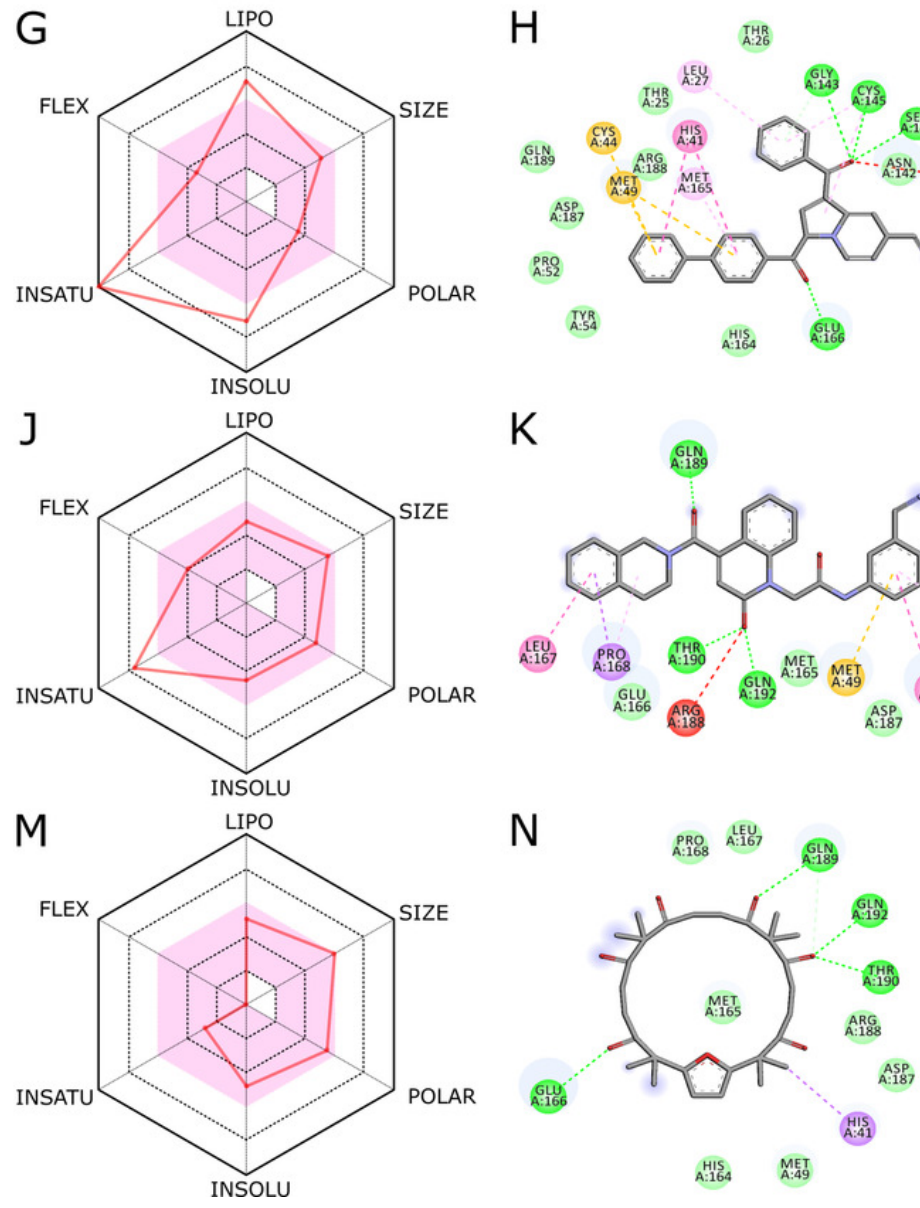
Figure 4

Spatial fluctuations of protein and ligands during molecular dynamics simulations of complexes

(A) C-alpha RMSD (running averages) for 3CL-PRO in complexes. (B) Ligand RMSD (running averages) in complexes. (C) C-alpha RMSF for 3CL-PRO in complexes. (C) C-alpha RMSF for 3CL-PRO in complexes. (D) Ligand RMSF in complexes. 
$\mathbf{A}$

Protein C-alpha RMSD

(Least squares fit to $\mathrm{C}$-alpha)
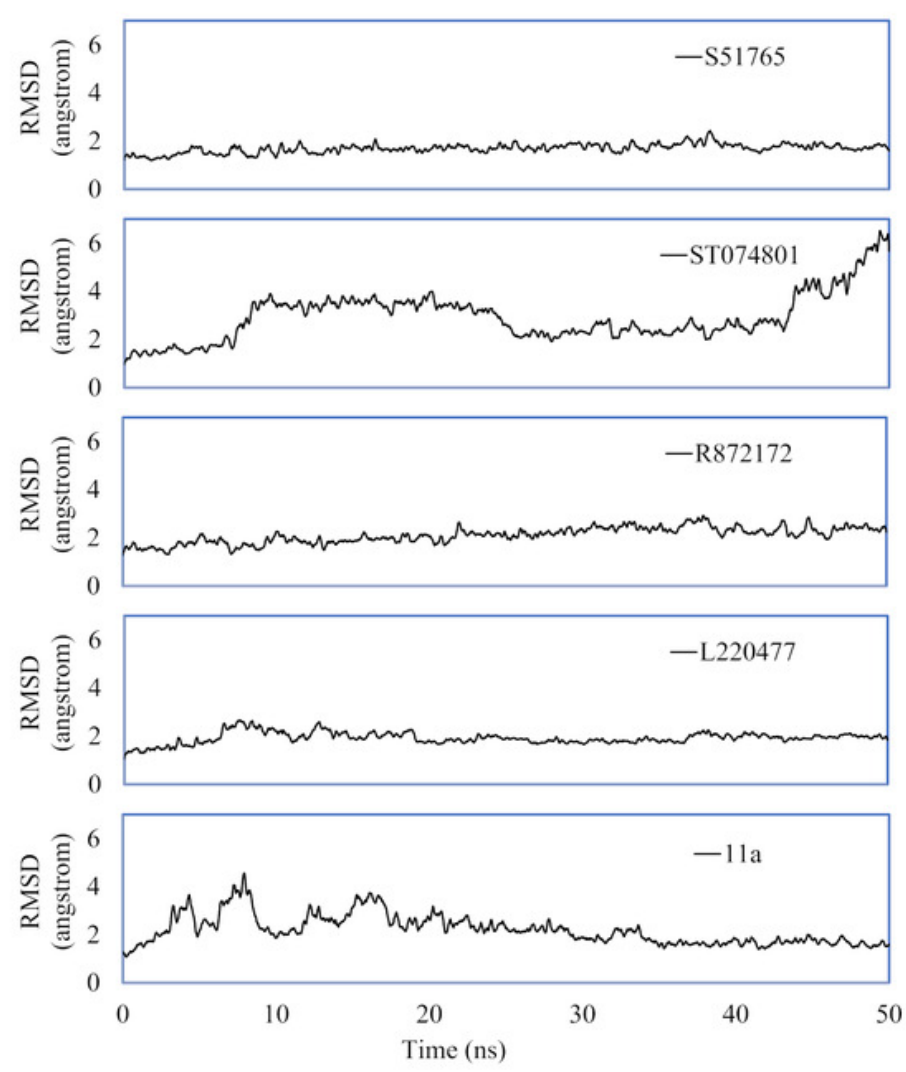

C

C-alpha RMSF

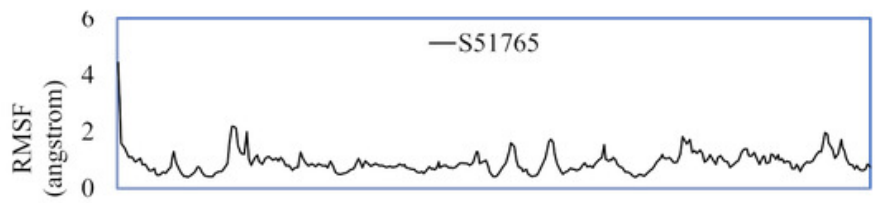

点

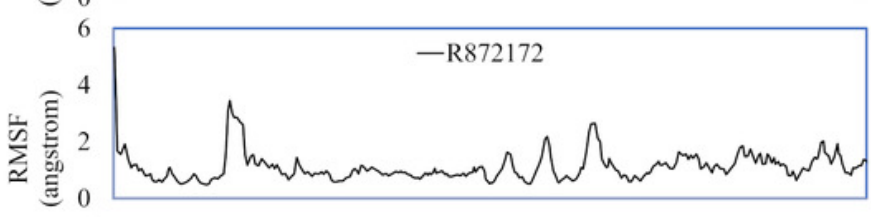

$6 \longdiv { \mathrm { L } 2 2 0 4 7 7 }$

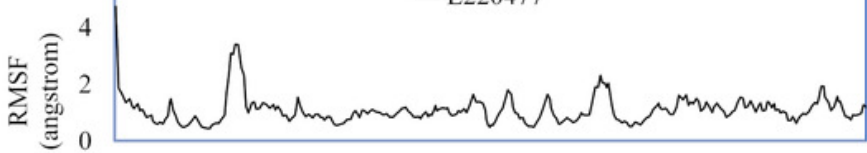

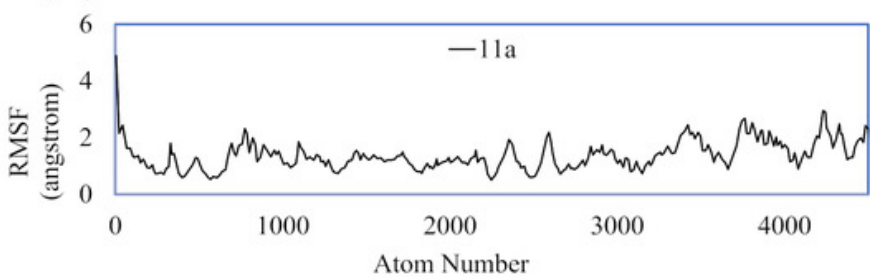

B
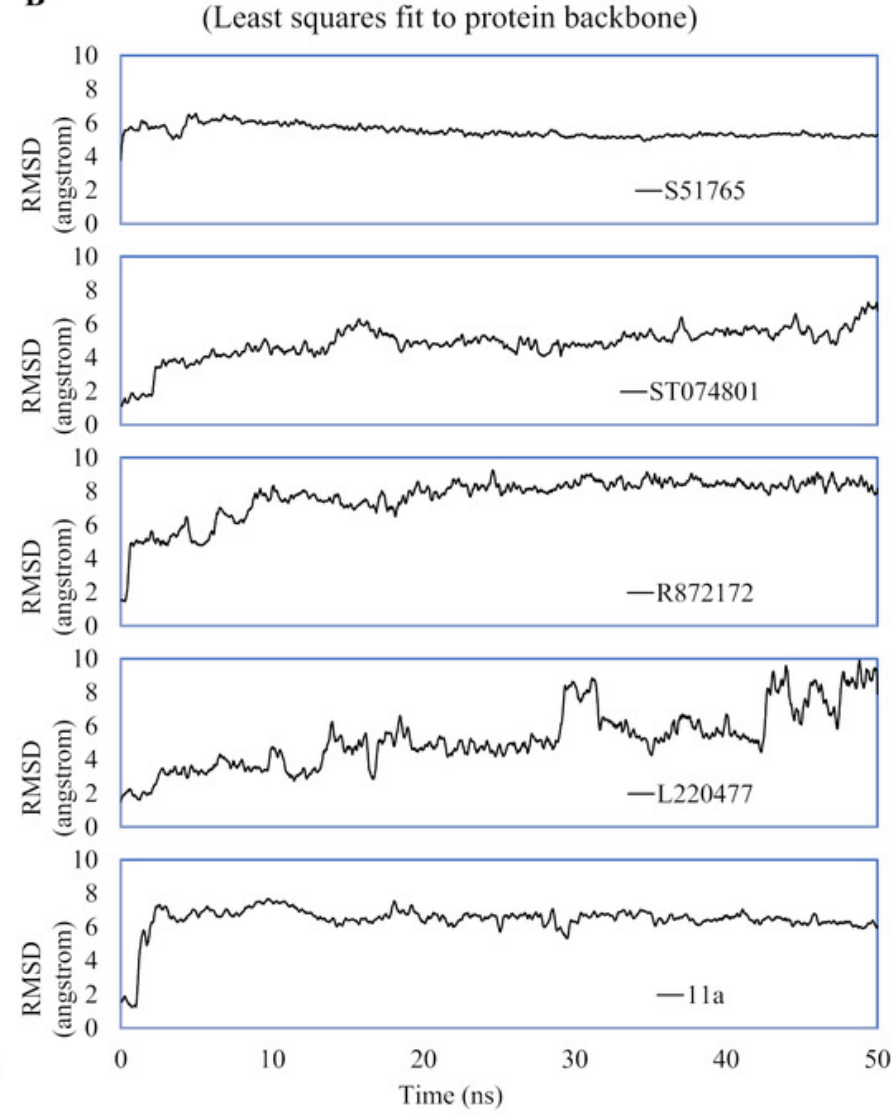

D

Ligand RMSF
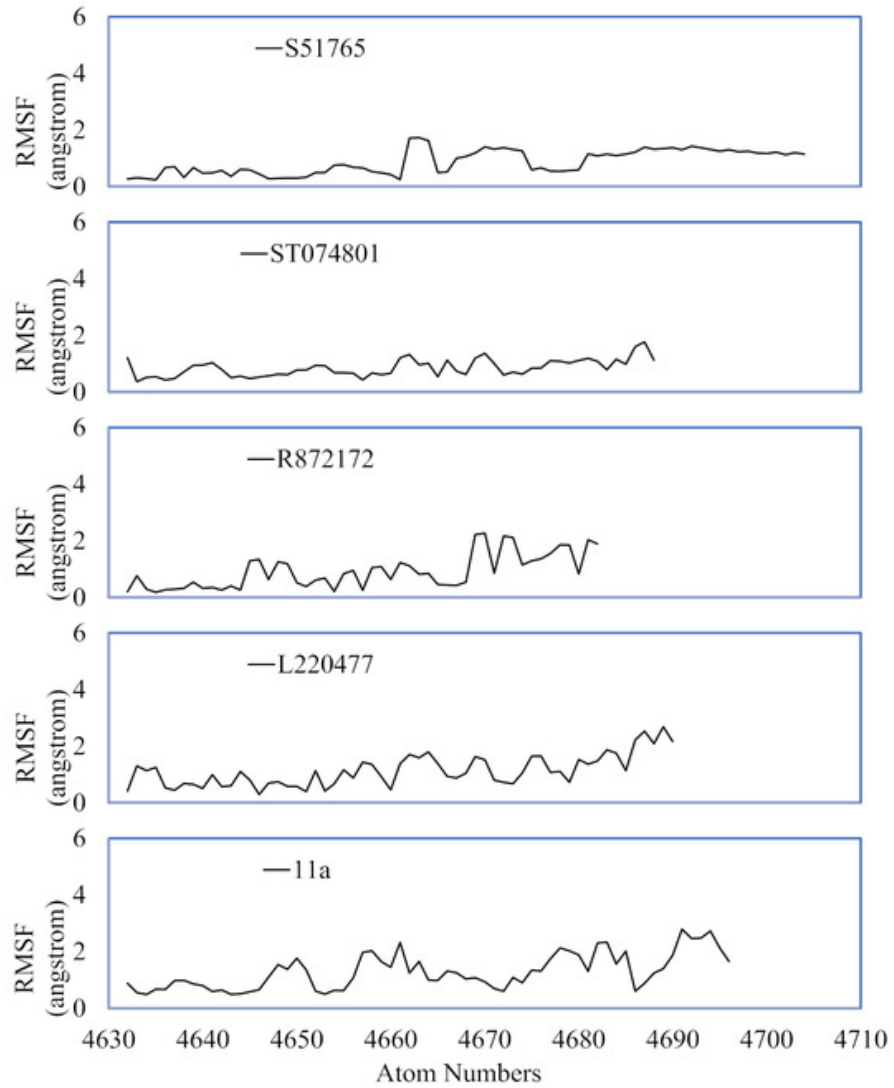
Figure 5

Protein-ligand binding modes in MD simulations of best ligands

Protein-ligand conformations at every $10 \mathrm{~ns}$ of simulation for 11a (A1-A6), L220477 (B1-B6), R872172 (C1-C6), ST074801 (D1-D6), and S517656 (E1-E6). 


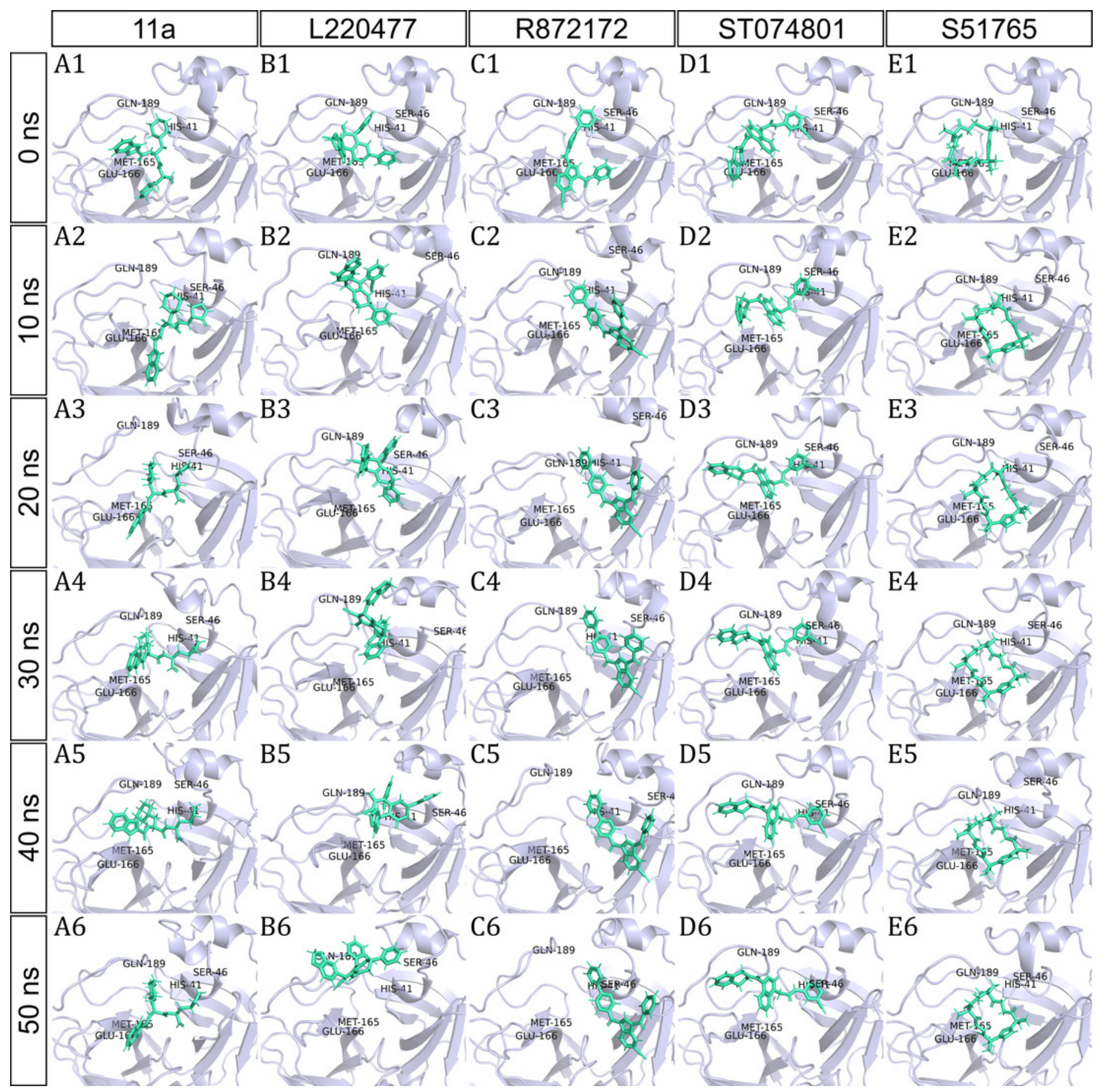




\section{Figure 6}

Analysis of hydrogen bonding interactions for best ligands.

(A-E) Number of hydrogen bonds between the ligand and 3CL-PRO during the simulation

period. (F) Occupancy of hydrogen bonding for the best ligands. (G) Occupancy of hydrogen bonding of the ligand with some important residues at the active site of 3CL-PRO. 

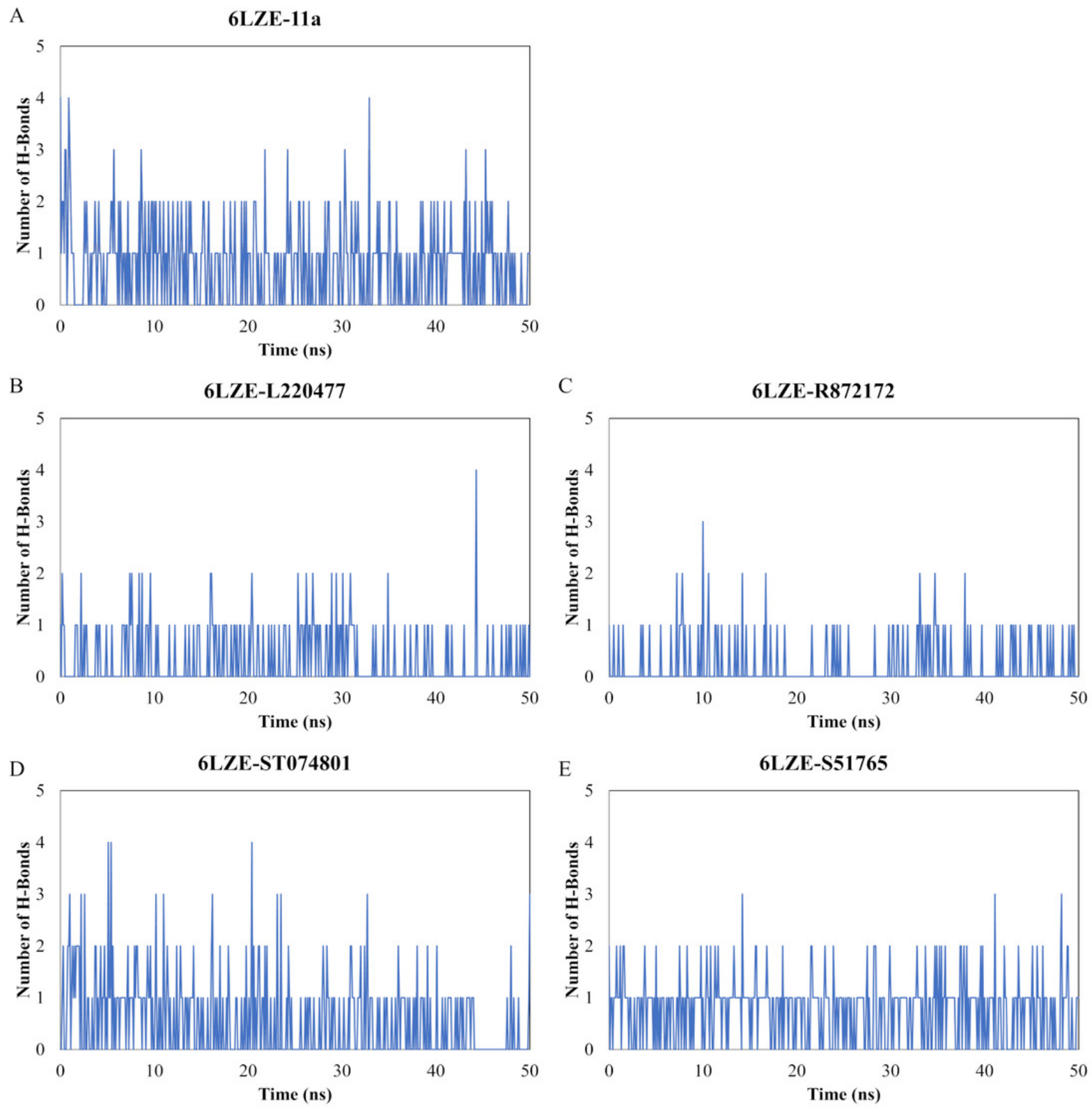

F
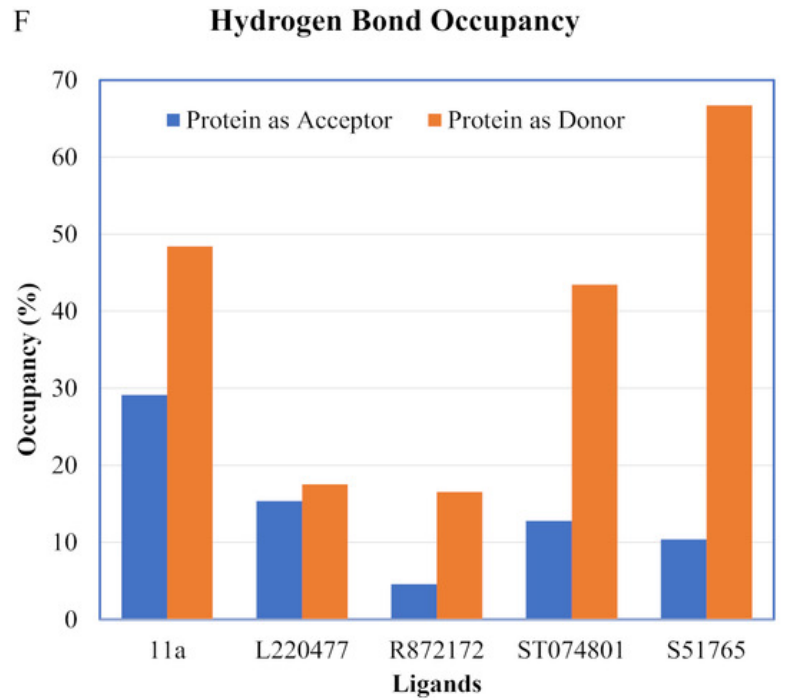

G Hydrogen Bond Occupancy by Residues (Protein as the donor)

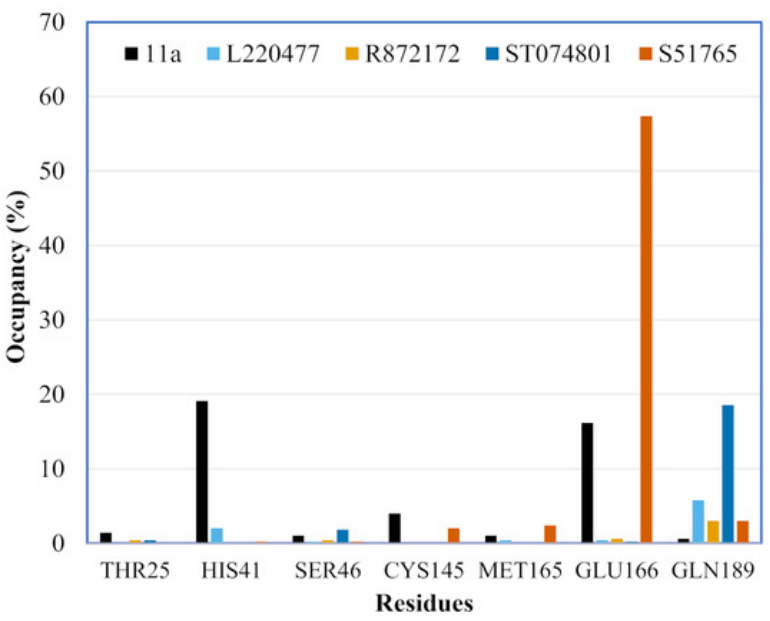


Figure 7

Key distances (running averages of 20 ps) between the ligand and the key amino acid residues of the target protein

Distances (in angstrom) are plotted against time for (A) 11a and HIS41, (B) L220477 and ASP187, (C) R872172, (D) ST074801, and (E) S51765.

\section{Distances between hydrogen-bond donor-acceptor atoms}

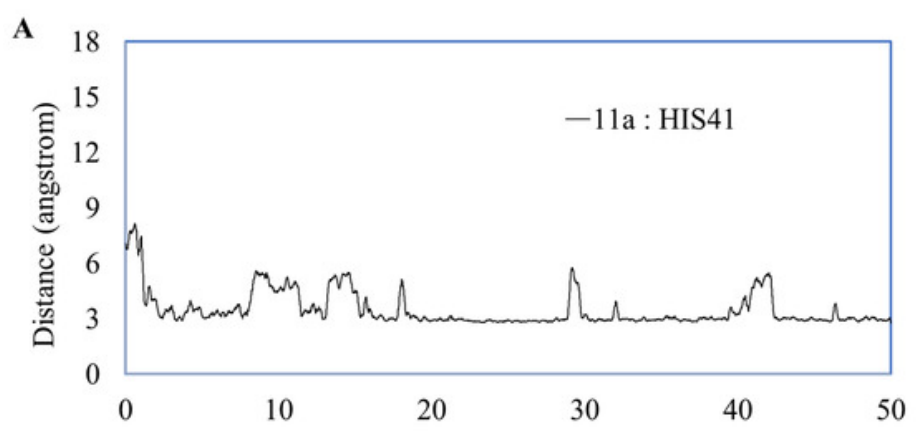

B
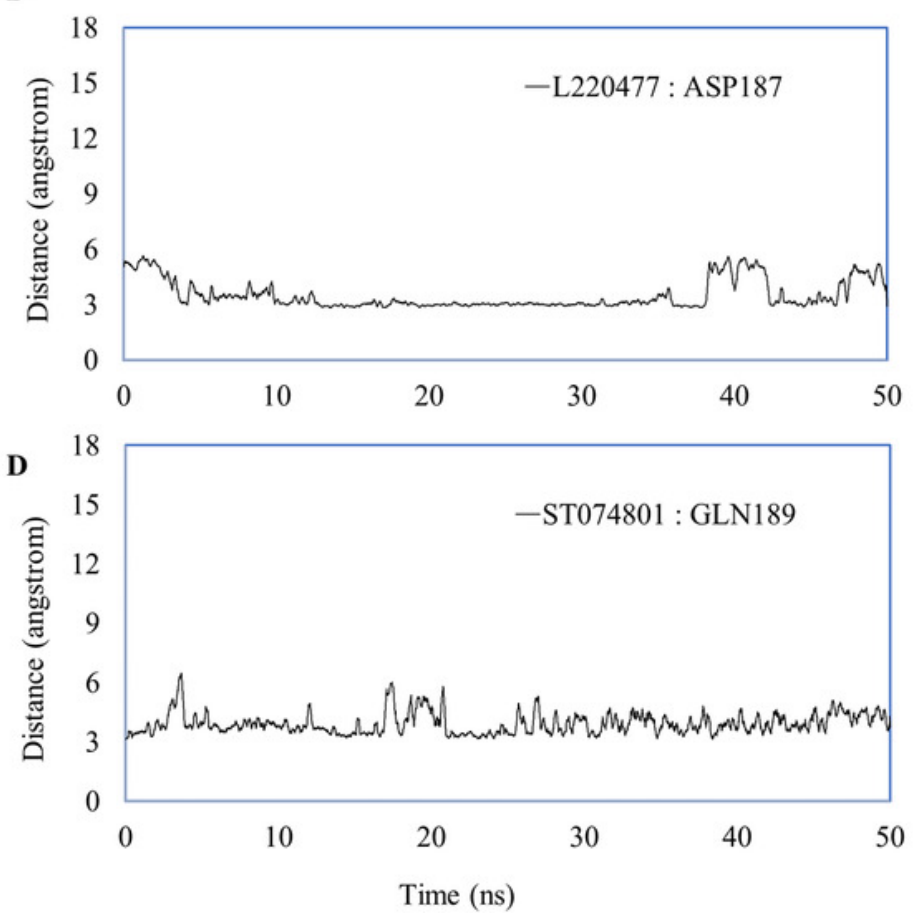

C
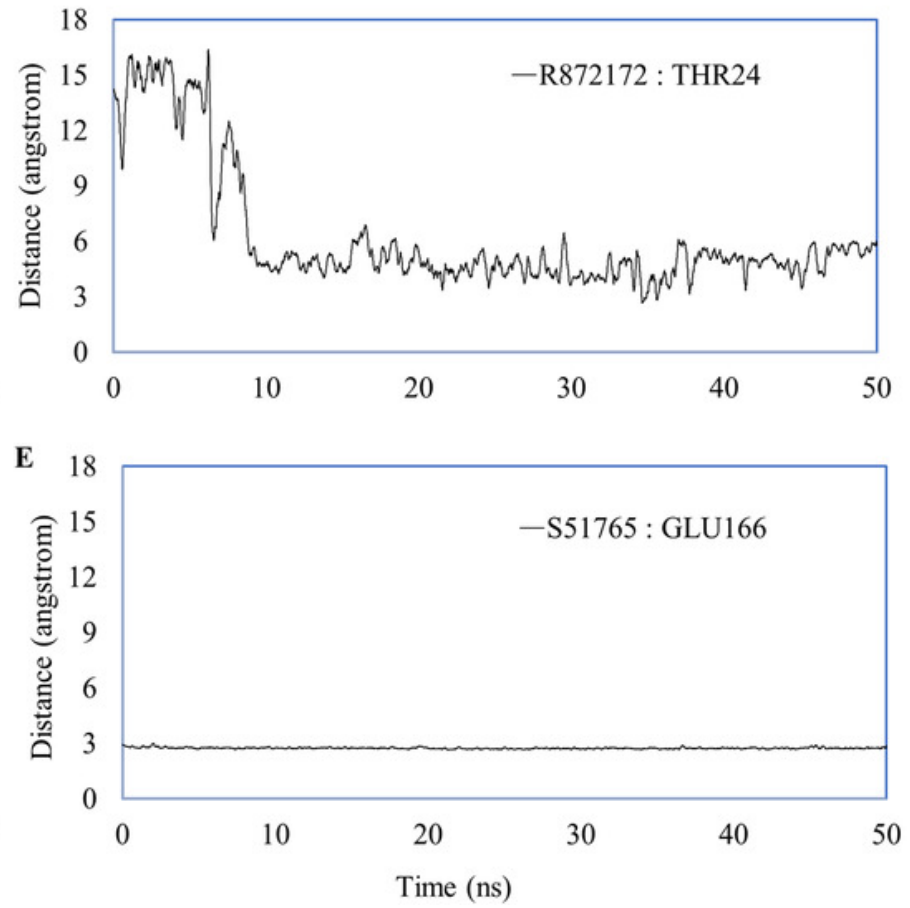
Figure 8

Protein-ligand interaction energies from molecular dynamics simulations for complexes of best ligands.

(A) Average short-range Coulomb (Coul-SR) and short-range Lennard-Jones (LJ-SR) energies for the complexes. Error bars show standard deviations. (B-F) Coul-SR and LJ-SR for the complexes over the simulation period. 
A

Protein-Ligand Interaction Energy

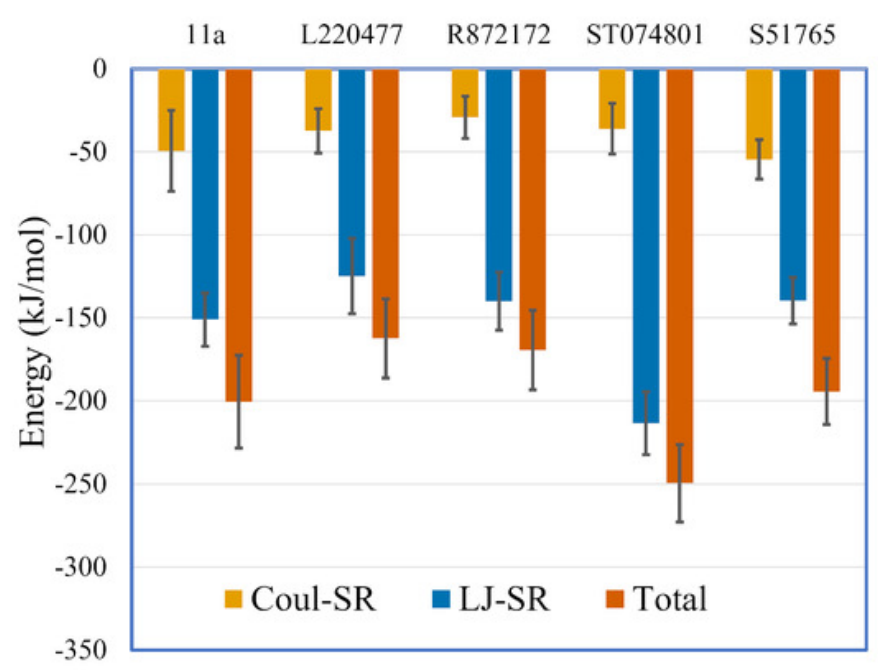

C

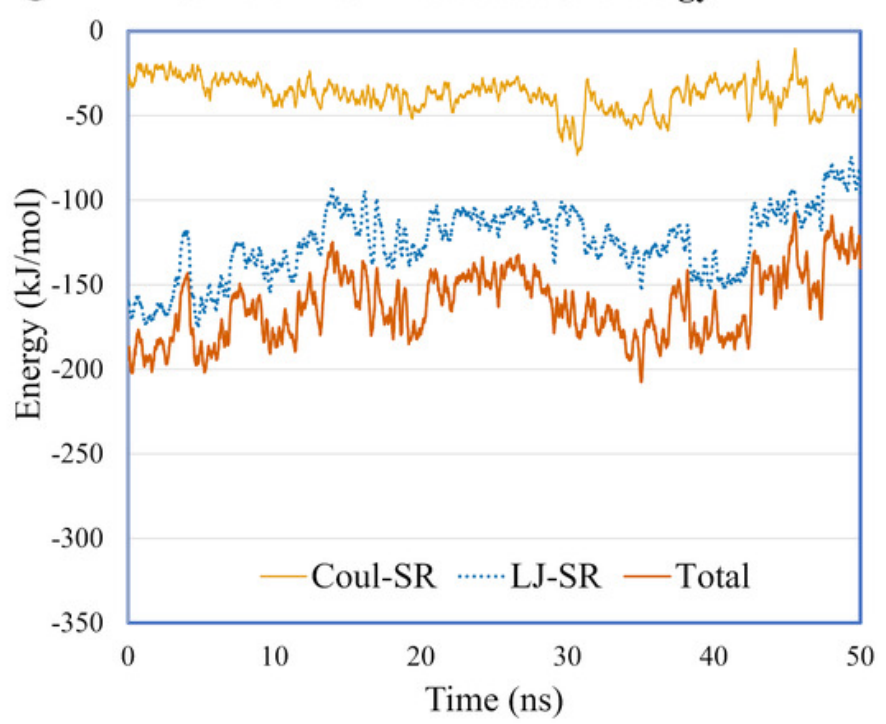

$\mathbf{E}$ 6LZE-ST074801 Interaction Energy

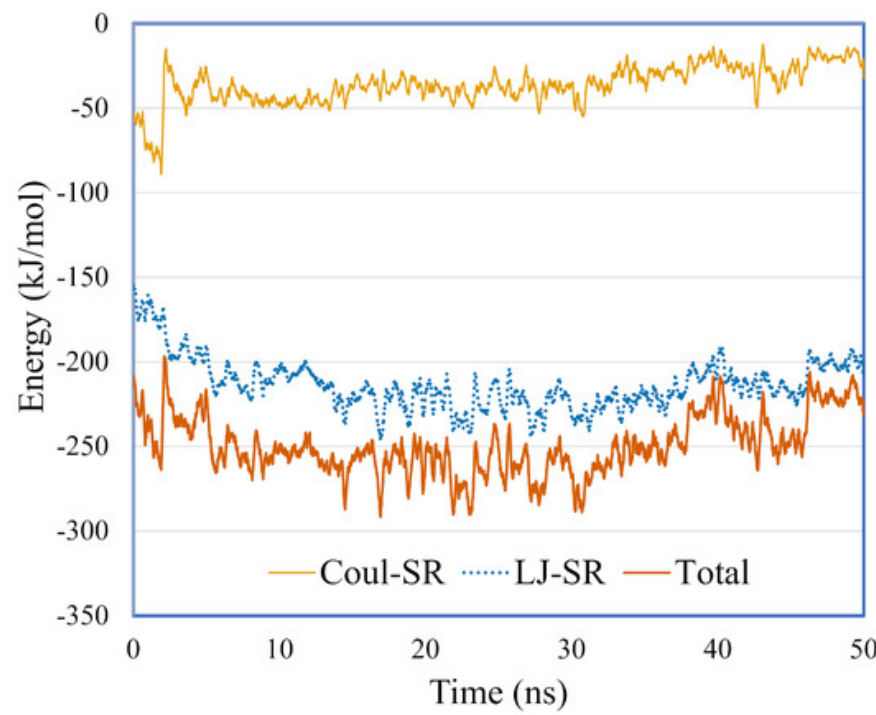

B

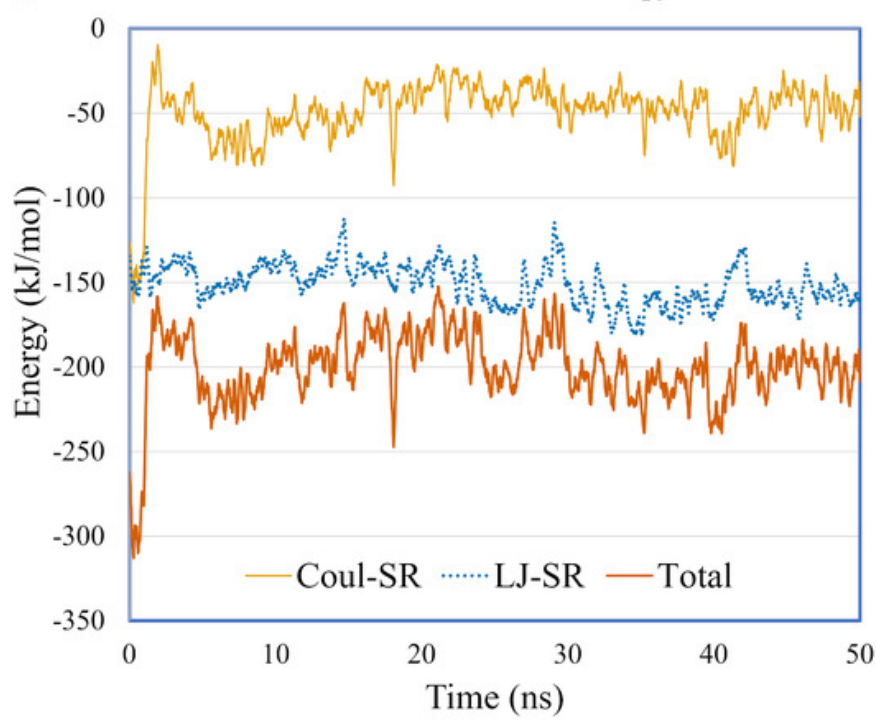

D

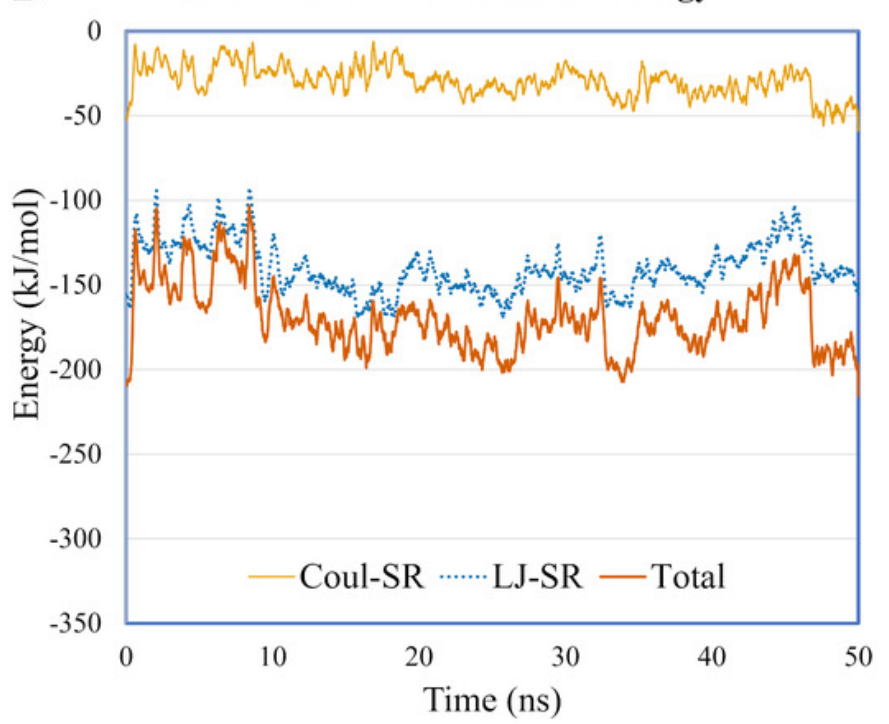

F

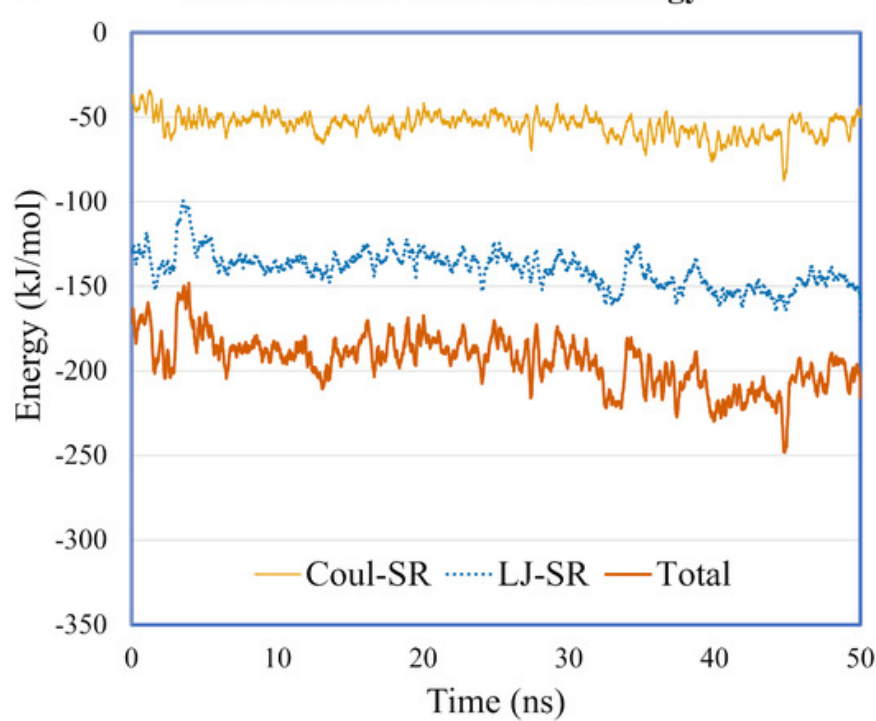




\section{Table $\mathbf{1}$ (on next page)}

Top ligands from the virtual screening of MyriaScreen Diversity Library II against 33 structures of 3CL-PRO. 


\begin{tabular}{|c|c|c|c|c|c|c|c|c|c|}
\hline \multirow[b]{3}{*}{ Rank } & \multirow[b]{3}{*}{ Ligand ID } & \multicolumn{8}{|c|}{ Predicted Binding Affinity (kcal/mol) } \\
\hline & & \multicolumn{2}{|c|}{ Total } & \multicolumn{3}{|c|}{ Single Crystal Structures } & \multicolumn{3}{|c|}{$\begin{array}{l}\text { MD Simulation Structures } \\
\text { (Average of ten structures) }\end{array}$} \\
\hline & & Average & SD & 6LZE & $6 \mathrm{M} 0 \mathrm{~K}$ & 6YB7 & 6LZE_MD & 6M0K_MD & 6BY7_MD \\
\hline 1 & R897698 & -8.7 & 0.5 & -9.7 & -9.0 & -8.3 & -8.8 & -8.3 & -8.9 \\
\hline 2 & ST031238 & -8.4 & 0.5 & -9.2 & -8.7 & -8.2 & -8.6 & -8.3 & -8.4 \\
\hline 3 & ST042014 & -8.4 & 0.6 & -9.8 & -9.3 & -8.2 & -8.6 & -7.9 & -8.3 \\
\hline 4 & ST018363 & -8.3 & 0.7 & -9.7 & -8.8 & -8.5 & -8.5 & -7.6 & -8.3 \\
\hline 5 & L363340 & -8.2 & 0.6 & -9.2 & -8.4 & -7.9 & -8.2 & -7.9 & -8.3 \\
\hline 6 & ST031351 & -8.1 & 0.5 & -8.7 & -7.9 & -9.3 & -8.3 & -7.6 & -8.2 \\
\hline 7 & L220477 & -8.1 & 0.8 & -9.4 & -8.7 & -8.0 & -8.4 & -7.2 & -8.4 \\
\hline 8 & R679445 & -8.1 & 0.6 & -8.8 & -8.5 & -8.5 & -8.3 & -7.5 & -8.3 \\
\hline 9 & ST000954 & -8.1 & 0.6 & -9.0 & -8.4 & -8.8 & -8.4 & -7.6 & -8.0 \\
\hline 10 & R872172 & -8.1 & 0.6 & -9.2 & -8.9 & -8.4 & -8.2 & -7.5 & -8.3 \\
\hline 11 & ST074801 & -8.1 & 0.5 & -8.9 & -8.0 & -8.1 & -8.3 & -7.6 & -8.3 \\
\hline 12 & ST088323 & -8.1 & 0.5 & -8.9 & -8.6 & -8.4 & -8.1 & -7.6 & -8.2 \\
\hline 13 & ST018407 & -8.1 & 0.6 & -8.8 & -8.7 & -8.8 & -8.2 & -7.9 & -7.9 \\
\hline 14 & S51765 & -8.0 & 0.6 & -8.6 & -8.2 & -8.6 & -8.2 & -7.5 & -8.1 \\
\hline 15 & ST074799 & -8.0 & 0.5 & -8.8 & -8.1 & -8.9 & -8.1 & -7.6 & -8.2 \\
\hline 16 & R818984 & -8.0 & 0.7 & -9.2 & -9.1 & -9.0 & -8.1 & -7.4 & -8.2 \\
\hline 17 & ST094780 & -8.0 & 0.6 & -8.6 & -8.2 & -8.3 & -8.3 & -7.3 & -8.1 \\
\hline 18 & ST020475 & -8.0 & 0.6 & -9.3 & -9.1 & -8.3 & -8.1 & -7.4 & -8.1 \\
\hline 19 & L128643 & -8.0 & 0.7 & -9.9 & -8.4 & -8.5 & -8.0 & -7.6 & -7.9 \\
\hline 20 & R461083 & -8.0 & 0.5 & -9.1 & -8.4 & -8.3 & -8.1 & -7.5 & -8.0 \\
\hline
\end{tabular}


Table 2 (on next page)

Computed physicochemical properties of top ligands. 


\begin{tabular}{|c|c|c|c|c|c|c|c|c|c|}
\hline & & \multicolumn{4}{|c|}{ Physicochemical Properties } & \multicolumn{2}{|c|}{ Lipid Solubility } & \multicolumn{2}{|c|}{ Water Solubility } \\
\hline Rank & Ligand ID & $\begin{array}{l}\text { \#Rotatable } \\
\text { bonds }\end{array}$ & $\begin{array}{l}\text { \#H-bond } \\
\text { acceptors }\end{array}$ & $\begin{array}{c}\text { \#H-bond } \\
\text { donors }\end{array}$ & TPSA & WLOGP & $\begin{array}{c}\text { Consensus } \\
\text { LogP }\end{array}$ & $\begin{array}{l}\text { ESOL } \\
\text { LogS }\end{array}$ & $\begin{array}{l}\text { ESOL } \\
\text { Class }\end{array}$ \\
\hline 1 & R897698 & 4 & 6 & 0 & 120.6 & 5.31 & 3.31 & -6.41 & $\begin{array}{l}\text { Poorly } \\
\text { soluble }\end{array}$ \\
\hline 2 & ST031238 & 5 & 7 & 1 & 149.68 & 1.57 & 1.45 & -4.26 & $\begin{array}{c}\text { Moderately } \\
\text { soluble }\end{array}$ \\
\hline 3 & ST042014 & 7 & 9 & 2 & 134.17 & 4.61 & 2.82 & -4.97 & $\begin{array}{c}\text { Moderately } \\
\text { soluble }\end{array}$ \\
\hline 4 & ST018363 & 5 & 8 & 1 & 108.37 & 6.54 & 4.11 & -6.46 & $\begin{array}{l}\text { Poorly } \\
\text { soluble }\end{array}$ \\
\hline 5 & L363340 & 4 & 5 & 0 & 101.2 & 5.88 & 4.92 & -6.5 & $\begin{array}{l}\text { Poorly } \\
\text { soluble }\end{array}$ \\
\hline 6 & ST031351 & 5 & 7 & 1 & 137.57 & 3.25 & 2.43 & -4.81 & $\begin{array}{c}\text { Moderately } \\
\text { soluble }\end{array}$ \\
\hline 7 & L220477 & 3 & 5 & 1 & 112.37 & 4.6 & 3.79 & -5.94 & $\begin{array}{c}\text { Moderately } \\
\text { soluble }\end{array}$ \\
\hline 8 & R679445 & 3 & 4 & 1 & 140.81 & 5.69 & 5.75 & -7.1 & $\begin{array}{l}\text { Poorly } \\
\text { soluble }\end{array}$ \\
\hline 9 & ST000954 & 5 & 6 & 4 & 141.12 & -1.51 & 0.79 & -3.77 & Soluble \\
\hline 10 & R872172 & 5 & 3 & 0 & 62.34 & 5.94 & 5.14 & -7.08 & $\begin{array}{l}\text { Poorly } \\
\text { soluble }\end{array}$ \\
\hline 11 & ST074801 & 6 & 4 & 1 & 95.2 & 2.99 & 3.09 & -4.57 & $\begin{array}{c}\text { Moderately } \\
\text { soluble }\end{array}$ \\
\hline 12 & ST088323 & 4 & 8 & 2 & 137.57 & 1.75 & 2.02 & -3.9 & Soluble \\
\hline 13 & ST018407 & 5 & 4 & 1 & 68.27 & 7.39 & 6.11 & -7.36 & $\begin{array}{l}\text { Poorly } \\
\text { soluble }\end{array}$ \\
\hline 14 & S51765 & 0 & 7 & 0 & 115.56 & 4.66 & 3.66 & -4.91 & $\begin{array}{c}\text { Moderately } \\
\text { soluble }\end{array}$ \\
\hline 15 & ST074799 & 6 & 3 & 1 & 71.41 & 3.73 & 4.02 & -5.23 & $\begin{array}{l}\text { Moderately } \\
\text { soluble }\end{array}$ \\
\hline 16 & R818984 & 4 & 3 & 0 & 51.44 & 6.1 & 5.29 & -7.57 & $\begin{array}{l}\text { Poorly } \\
\text { soluble }\end{array}$ \\
\hline 17 & ST094780 & 3 & 5 & 1 & 69.72 & 3.04 & 3.59 & -5.23 & $\begin{array}{l}\text { Moderately } \\
\text { soluble }\end{array}$ \\
\hline 18 & ST020475 & 4 & 5 & 2 & 99.85 & 2.89 & 2.98 & -4.9 & $\begin{array}{c}\text { Moderately } \\
\text { soluble }\end{array}$ \\
\hline 19 & L128643 & 1 & 3 & 0 & 29.54 & 5.39 & 5.02 & -6.39 & $\begin{array}{l}\text { Poorly } \\
\text { soluble }\end{array}$ \\
\hline 20 & R461083 & 2 & 3 & 2 & 59.59 & 3.93 & 3.82 & -5.59 & $\begin{array}{l}\text { Moderately } \\
\text { soluble }\end{array}$ \\
\hline
\end{tabular}




\section{Table 3(on next page)}

Predicted metabolic and elimination profiles of top ligands. 


\begin{tabular}{|c|c|c|c|c|c|c|c|c|}
\hline & \multicolumn{3}{|c|}{ Inhibitor } & \multicolumn{2}{c|}{ Elimination } \\
\hline Rank & Ligand ID & CYP1A2 & CYP2C19 & CYP2C9 & CYP2D6 & CYP3A4 & $T_{1 / 2}(\mathrm{~h})$ & $\begin{array}{c}\text { Clearance } \\
\text { (ml/min/kg) }\end{array}$ \\
\hline 1 & R897698 & No & Yes & Yes & No & Yes & 1.825 & 0.749 \\
\hline 2 & ST031238 & No & Yes & Yes & No & No & 1.81 & 0.83 \\
\hline 3 & ST042014 & No & No & Yes & No & No & 1.71 & 0.44 \\
\hline 4 & ST018363 & No & Yes & Yes & No & Yes & 1.83 & 1.01 \\
\hline 5 & L363340 & Yes & Yes & Yes & No & No & 1.7 & 1.48 \\
\hline 6 & ST031351 & No & Yes & Yes & No & No & 1.61 & 0.8 \\
\hline 7 & L220477 & No & Yes & Yes & No & Yes & 2.05 & 1.53 \\
\hline 8 & R679445 & No & Yes & Yes & No & No & 2.03 & 0.91 \\
\hline 9 & ST000954 & No & No & No & No & No & 1.78 & 0.8 \\
\hline 10 & R872172 & Yes & Yes & Yes & No & No & 1.98 & 1.37 \\
\hline 11 & ST074801 & No & Yes & Yes & No & Yes & 1.94 & 1.3 \\
\hline 12 & ST088323 & No & No & No & No & No & 0.99 & 0.75 \\
\hline 13 & ST018407 & No & Yes & No & No & No & 1.87 & 1.52 \\
\hline 14 & S51765 & No & No & No & No & Yes & 1.94 & 1.27 \\
\hline 15 & ST074799 & No & Yes & Yes & No & Yes & 2.07 & 1.35 \\
\hline 16 & R818984 & Yes & Yes & No & No & No & 2.21 & 1.25 \\
\hline 17 & ST094780 & No & Yes & Yes & Yes & Yes & 1.65 & 1.16 \\
\hline 18 & ST020475 & Yes & Yes & Yes & No & No & 1.37 & 0.78 \\
\hline 19 & L128643 & Yes & Yes & No & No & No & 2.11 & 1.44 \\
\hline 20 & R461083 & Yes & Yes & Yes & Yes & Yes & 2.06 & 1.82 \\
\hline
\end{tabular}


Table 4 (on next page)

Druglikeness of top ligands 


\begin{tabular}{|c|c|c|c|c|c|c|}
\hline Rank & Ligand ID & Lipinski & Ghose & Veber & Egan & Muegge \\
\hline 1 & R897698 & Yes & No & Yes & Yes & No \\
\hline 2 & ST031238 & Yes & Yes & No & No & Yes \\
\hline 3 & ST042014 & Yes & No & Yes & No & Yes \\
\hline 4 & ST018363 & Yes & No & Yes & No & No \\
\hline 5 & L363340 & Yes & No & Yes & Yes & No \\
\hline 6 & ST031351 & Yes & Yes & Yes & No & Yes \\
\hline 7 & L220477 & Yes & No & Yes & Yes & Yes \\
\hline 8 & R679445 & Yes & No & No & No & No \\
\hline 9 & ST000954 & Yes & No & No & No & Yes \\
\hline 10 & R872172 & Yes & No & Yes & No & No \\
\hline 11 & ST074801 & Yes & No & Yes & Yes & Yes \\
\hline 12 & ST088323 & Yes & Yes & Yes & No & Yes \\
\hline 13 & ST018407 & Yes & No & Yes & No & No \\
\hline 14 & S51765 & Yes & No & Yes & Yes & Yes \\
\hline 15 & ST074799 & Yes & No & Yes & Yes & Yes \\
\hline 16 & R818984 & Yes & No & Yes & No & No \\
\hline 17 & ST094780 & Yes & No & Yes & Yes & Yes \\
\hline 18 & ST020475 & Yes & Yes & Yes & Yes & Yes \\
\hline 19 & L128643 & Yes & Yes & Yes & Yes & No \\
\hline 20 & R461083 & Yes & Yes & Yes & Yes & Yes \\
\hline
\end{tabular}


Table 5 (on next page)

Toxicity profiles of top ligands 


\begin{tabular}{|c|c|c|c|c|c|c|c|c|}
\hline & & \multicolumn{3}{|c|}{ ADMETlab } & \multicolumn{4}{|c|}{ OSIRIS } \\
\hline Rank & Ligand ID & $\begin{array}{l}\text { hERG } \\
\text { Blocker }\end{array}$ & $\begin{array}{c}\text { Hepatotoxic } \\
\text { ity }\end{array}$ & $\begin{array}{c}\text { Ames } \\
\text { Mutagenici } \\
\text { ty }\end{array}$ & $\begin{array}{l}\text { Mutagenes } \\
\text { is }\end{array}$ & $\begin{array}{c}\text { Tumorigene } \\
\text { sis }\end{array}$ & Irritant & $\begin{array}{l}\text { Reproductiv } \\
\text { e Effect }\end{array}$ \\
\hline 1 & R897698 & Medium & Low & No & Medium & High & No & No \\
\hline 2 & ST031238 & Low & Low & High & No & No & No & No \\
\hline 3 & ST042014 & Medium & Medium & No & High & High & No & No \\
\hline 4 & ST018363 & Low & Low & No & No & No & Medium & No \\
\hline 5 & L363340 & Medium & Low & No & No & No & No & No \\
\hline 6 & ST031351 & Low & Medium & High & No & No & No & No \\
\hline 7 & L220477 & Medium & Medium & No & No & No & No & Medium \\
\hline 8 & R679445 & Medium & Low & No & No & No & No & No \\
\hline 9 & ST000954 & Low & No & No & No & No & No & High \\
\hline 10 & R872172 & Medium & Low & Low & No & No & No & No \\
\hline 11 & ST074801 & Medium & Low & No & No & No & No & No \\
\hline 12 & ST088323 & Low & High & Low & High & High & No & Medium \\
\hline 13 & ST018407 & Medium & Medium & No & No & No & Medium & No \\
\hline 14 & S51765 & No & No & No & No & No & No & No \\
\hline 15 & ST074799 & Medium & Low & No & No & No & No & Medium \\
\hline 16 & R818984 & Medium & Low & Low & No & High & No & No \\
\hline 17 & ST094780 & Medium & Low & No & No & No & No & No \\
\hline 18 & ST020475 & Medium & High & No & High & High & No & No \\
\hline 19 & L128643 & Medium & Low & Low & High & High & No & No \\
\hline 20 & R461083 & Medium & Medium & No & No & No & No & No \\
\hline
\end{tabular}




\section{Table 6(on next page)}

Distances between the ligand and the key amino acid residues forming high-occupancy hydrogen bonds 


\begin{tabular}{|c|c|c|c|c|c|}
\hline Ligand & Donor & Acceptor & Occupancy & $\begin{array}{c}\text { Average Distance } \\
\text { (nm) }\end{array}$ & $\begin{array}{c}\text { Standard Deviation } \\
\text { (nm) }\end{array}$ \\
\hline 11a & HIS41 & $11 \mathrm{a}$ & $12.15 \%$ & 0.3494 & 0.10415 \\
\hline L220477 & L220477 & ASP187 & $5.18 \%$ & 0.35085 & 0.0853 \\
\hline R872172 & THR24 & R872172 & $2.19 \%$ & 0.62455 & 0.34955 \\
\hline ST074801 & GLN189 & ST074801 & $4.18 \%$ & 0.39249 & 0.08995 \\
\hline S51765 & GLU166 & S51765 & $55.38 \%$ & 0.27349 & 0.01284 \\
\hline S51765 (Simulation-2) & GLN189 & S51765 & $7.77 \%$ & 0.4569 & 0.14613 \\
\hline S51765 (Simulation-3) & GLU166 & S51765 & $57.97 \%$ & 0.29025 & 0.0559 \\
\hline S51765 (Simulation-4) & GLU166 & S51765 & $37.65 \%$ & 0.3124 & 0.06403 \\
\hline S51765 (Simulation-5) & GLU166 & S51765 & $49.00 \%$ & 0.29039 & 0.02189 \\
\hline
\end{tabular}




\section{Table 7 (on next page)}

Free energy calculations for best two ligands and the reference ligand 


\begin{tabular}{|c|c|c|c|c|c|c|}
\hline \multirow{2}{*}{ Energy Terms } & \multicolumn{3}{|c|}{ 11a:3CL-PRO Complex } & \multicolumn{3}{c|}{ S51765:3CL-PRO Complex } \\
\cline { 2 - 7 } & \multicolumn{3}{|c|}{ Simulation Period } & \multicolumn{3}{c|}{ Simulation Period } \\
\cline { 2 - 7 } & $20-25 \mathrm{~ns}$ & $45-50 \mathrm{~ns}$ & Mean & $20-25 \mathrm{~ns}$ & $45-50 \mathrm{~ns}$ & Mean \\
\hline van der Waal energy $(\mathrm{kJ} / \mathrm{mol})$ & -164.80 & -175.54 & -170.17 & -152.02 & -166.60 & -159.31 \\
\hline Electrostatic energy $(\mathrm{kJ} / \mathrm{mol})$ & -22.72 & -23.98 & -23.35 & -30.69 & -34.77 & -32.73 \\
\hline Polar solvation energy $(\mathrm{kJ} / \mathrm{mol})$ & 140.25 & 140.87 & 140.56 & 142.61 & 139.12 & 140.87 \\
\hline SASA energy $(\mathrm{kJ} / \mathrm{mol})$ & -19.55 & -20.45 & -20.00 & -19.02 & -18.82 & -18.92 \\
\hline Binding energy $(\mathrm{kJ} / \mathrm{mol})$ & -66.81 & -79.09 & -72.95 & -59.12 & -81.08 & -70.10 \\
\hline
\end{tabular}

1 